\title{
Review Article \\ Systematic Literature Review of the Epidemiology of Nongenetic Forms of Hypogonadism in Adult Males
}

\author{
Victoria Zarotsky, ${ }^{1}$ Ming-Yi Huang, ${ }^{2,3}$ Wendy Carman, ${ }^{4}$ Abraham Morgentaler, ${ }^{5,6}$ \\ Puneet K. Singhal, ${ }^{2}$ Donna Coffin, ${ }^{7}$ and T. Hugh Jones ${ }^{8,9}$ \\ ${ }^{1}$ Product Value Strategy Consulting, Optum, Eden Prairie, MN 55344, USA \\ ${ }^{2}$ Global Health Outcomes, Merck \& Co., Inc., West Point, PA 19486, USA \\ ${ }^{3}$ School of Pharmacy, Temple University, Philadelphia, PA 19140, USA \\ ${ }^{4}$ Epidemiology, Optum, Ann Arbor, MI 48108, USA \\ ${ }^{5}$ Men's Health Boston, Brookline, MA 02445, USA \\ ${ }^{6}$ Harvard Medical School, Boston, MA 02115, USA \\ ${ }^{7}$ Boolean Research Consulting, Westmount, QC, Canada \\ ${ }^{8}$ Robert Hague Centre for Diabetes and Endocrinology, Barnsley Hospital NHS Foundation Trust, Barnsley S752EP, UK \\ ${ }^{9}$ Department of Human Metabolism, School of Medicine and Biomedical Sciences, University of Sheffield, Sheffield S10 2RX, UK
}

Correspondence should be addressed to Victoria Zarotsky; victoria.zarotsky@optum.com

Received 20 May 2014; Accepted 23 June 2014; Published 22 July 2014

Academic Editor: Jack van Honk

Copyright ( 2014 Victoria Zarotsky et al. This is an open access article distributed under the Creative Commons Attribution License, which permits unrestricted use, distribution, and reproduction in any medium, provided the original work is properly cited.

\begin{abstract}
This study summarizes the literature on the prevalence, incidence, and proportion of patients receiving treatment for male hypogonadism and a systematic literature search was performed for articles published in the last 20 years. Of the 97 studies identified, 96 examined the prevalence, 2 examined the incidence, and 4 examined the proportion of males with hypogonadism patients receiving treatment. Based on studies conducted in Europe and USA, the prevalence of hypogonadism in the general population ranged from $2.1 \%$ to $12.8 \%$ of middle-aged to older men, with an estimated incidence of 12 new cases per 1,000 personyears. Prevalence was higher among patients with comorbid conditions, such as type 2 diabetes mellitus and obesity. Approximately $10-12 \%$ of men with hypogonadism were receiving testosterone treatment. This literature review suggests that there is potentially a significant burden of hypogonadism in the general population. Burden seems to increase with age and in the presence of certain disease conditions. Data suggests that many hypogonadal men who may benefit from testosterone replacement are not receiving treatment. This may be the result of underdiagnosis of the disease, lack of awareness by patients or physicians, irregularities surrounding the diagnostic criteria, and deficiency of long-term safety studies.
\end{abstract}

\section{Introduction}

Hypogonadism in men has been defined as a clinical syndrome resulting from failure of the testis to produce physiological levels of testosterone (androgen deficiency) and a normal number of spermatozoa, due to disruption of one or more levels of the hypothalamic-pituitary-testicular axis [1]. A diagnosis of hypogonadism is typically based on the signs and symptoms associated with low $\mathrm{T}$, followed by biochemical confirmation of low testosterone (T) [1]. The most widely accepted parameter used to establish hypogonadism is the measurement of serum total testosterone (TT) [1]; however, cut-off values used to indicate hypogonadism have not been clearly defined and vary across studies. Recent clinical practice guidelines published by the Endocrine Society have reported that the average TT threshold, at which the likelihood of most symptoms associated with hypogonadism increases, corresponds to the lower limit of the normal range for young men, that is, approximately $300 \mathrm{ng} / \mathrm{dL}$ (10.4 nmol/liter) [1]. Correspondingly, a common threshold 
TABLE 1: Search strategy executed in PubMed/MEDLINE, EMBASE, and The Cochrane Central Register of Controlled Trials August 31, 2012, and incidence, prevalence, and treatment rates.

\begin{tabular}{|c|c|c|}
\hline Search & Search string [field] & Hits \\
\hline 1 & Search "hypogonadism/epidemiology" [mesh] & 345 \\
\hline 2 & $\begin{array}{l}\text { Search hypogonadism [title] OR testosterone deficiency* [title] OR "testosterone/deficiency" [mesh] OR } \\
\text { testoid deficiency* [title] OR androgen deficiency* [title] OR testosterone replacement therapy* [title] }\end{array}$ & 3173 \\
\hline 3 & $\begin{array}{l}\text { Search epidemiology* [title/abstract] OR incidence [title/abstract] OR prevalence [title/abstract] OR rate } \\
\text { [title/abstract] OR rates [title/abstract] OR treatment rate [title/abstract] OR treatment rates [title/abstract] }\end{array}$ & 2380843 \\
\hline 4 & Search (“epidemiology” [mesh]) OR “incidence” [mesh] OR "prevalence” [mesh]) & 318545 \\
\hline 5 & Search \#3 OR \#4 & 2472821 \\
\hline 6 & Search \#2 AND \#5 & 331 \\
\hline 7 & Search \#1 OR \#6 & 603 \\
\hline \multirow[t]{2}{*}{8} & Search \#7 filters: publication date from 1992/01/01 to 2012/8/31; humans; English; male; adult: $19+$ years & 298 \\
\hline & Non-English articles & 21 \\
\hline
\end{tabular}

used in the literature to indicate hypogonadism is serum TT $<300 \mathrm{ng} / \mathrm{dL}(<10.4 \mathrm{nmol} / \mathrm{L})$; however, cut-off values of $<200 \mathrm{ng} / \mathrm{dL}(6.94 \mathrm{nmol} / \mathrm{L})$ to $<350 \mathrm{ng} / \mathrm{dL}(<12 \mathrm{nmol} / \mathrm{L})$ are not uncommon. These thresholds are sometimes combined with requisite signs and symptoms, but not always. The result is a range of operational definitions of hypogonadism used in the literature, varying in degree of stringency and potentially leading to variations in estimates of prevalence of the disease.

The literature on the epidemiology of hypogonadism is confounded by considerable heterogeneity across studies, varying not only by operational definition but also by patient populations, study design, time frame of study, and geography. Estimates of prevalence, incidence, and treatment rates are influenced by all of these factors. The aim of this systematic review of the epidemiology of hypogonadism was to determine the prevalence and incidence of hypogonadism, as well as the proportion of males with hypogonadism receiving treatment of hypogonadism, while accounting for differences in patient and study characteristics and operational definition of hypogonadism used.

\section{Methods}

2.1. Eligibility Criteria. Eligible studies were defined in this systematic review as original studies that assessed the prevalence, incidence, or treatment rates of adult males $(\geq 18$ years of age) with hypogonadism, acquired or idiopathic. Studies on genetic forms of hypogonadism (i.e., Klinefelter's syndrome) and secondary publications of the same results were not included. Observational studies with sample sizes $>30$ were included in this review; treatment and intervention studies were excluded.

2.2. Search Methods for Identification of Studies. Three pharmacologic and biomedical literature databases were searched for this systematic literature review: PubMed/MEDLINE database, Cochrane Library Database, and EMBASE. Search strings composed of medical subject heading (MeSH) terms, from the controlled vocabulary indexing hierarchy and/or intext terms in title and/or abstract fields, were used (Table 1).
Citations were limited to English language articles, published during a 20-year period (January 01, 1992, to August 31, 2012). The reference list of included publications was searched for relevant articles.

2.3. Data Extraction. We abstracted key data elements using a standardized abstraction tool, including type of research design, country, study period, sample size, operational definition of hypogonadism used, study population characteristics, and outcomes. One author screened the search results against the predefined inclusion and exclusion criteria. Titles were evaluated, abstracts were reviewed, and potentially relevant full-text articles were evaluated for relevance. A quality check was conducted on $20 \%$ of the abstracts by a second reviewer.

We categorized studies based on population of interest: population-based studies were those with large sample sizes $(>1$ thousand) and minimal age restrictions and conducted in a specific geographical location; community-based studies were those with relatively smaller sample sizes (i.e., hundreds) and more restricted age ranges and conducted in a specific geographical location; primary care-based studies were those that recruited patients from a general primary care or medical clinic seeking medical care; screening-based studies recruited healthy individuals from a general health screening clinic. Primary care-based studies and screeningbased studies were classified together as they were conducted in the same setting. Finally, clinical condition-based studies were those that examined prevalence of hypogonadism in patient populations with specific clinical conditions. We also separately summarized prevalence of hypogonadism by age for studies that provided these data.

\section{Results}

3.1. Literature Search Results. The literature search identified 1,395 articles that were reviewed for inclusion. After review of titles and abstracts, we evaluated 175 full-text articles and identified 97 articles that met our eligibility criteria: 96 reported on the prevalence of hypogonadism, 2 on the incidence, and 4 on treatment proportions (not mutually 


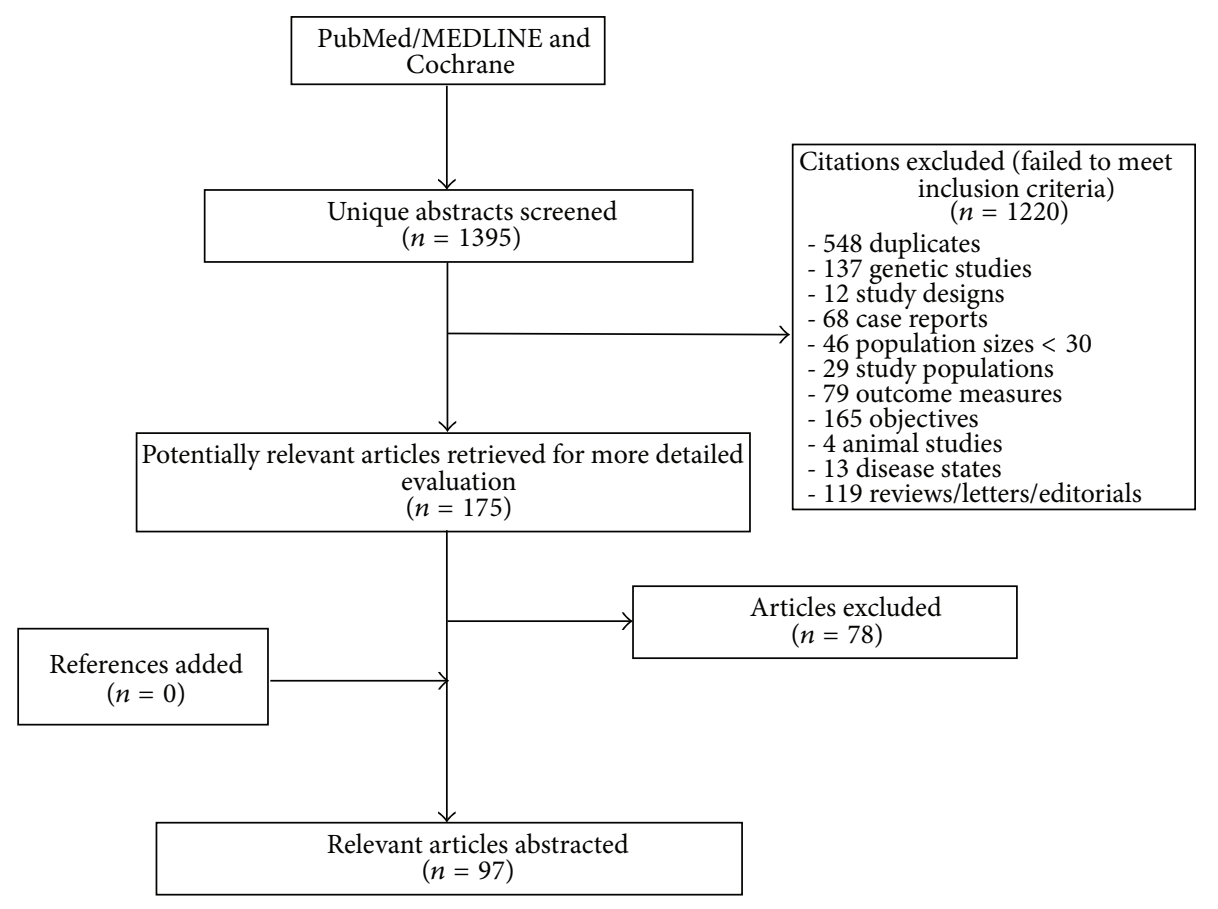

Figure 1: Flow diagram.

exclusive). The PRISMA (preferred reporting items for systematic reviews and meta-analyses) flow chart can be found in Figure 1.

3.2. Prevalence of Hypogonadism. Among the 96 identified prevalence studies, 7 were population- $(n=4)$ [2-5] or community- $(n=3)$ [6-8] based studies and 7 were primary care or health screening studies [9-15]. Ten examined agerelated changes for hypogonadism [2, 3, 8, 9, 12, 13, 16-19] and seventy-eight were clinical condition-based studies [20-97]. Studies varied considerably in geographic location, patient characteristics, sample sizes, and operational definition for hypogonadism. These studies are described in Tables 2 and 3.

3.2.1. Population-Based Studies. The prevalence of hypogonadism in the 4 population-based studies ranged from $2.1 \%$ to $12.8 \%$ [2-5] (Table 2). Sample sizes in these studies ranged from 1,490 to 2,966 participants and the mean (SD) ages of the study participants ranged from 47.3 (12.5) to 59.15 (10.8) years. Mean age was not reported in one of the studies [3]; however, the sample was equally divided among men in their $40 \mathrm{~s}, 50 \mathrm{~s}$, and 60s [3]. The studies were conducted in Europe (Belgium, Estonia, Hungary, Italy, Poland, Spain, Sweden, and UK) and USA.

The operational definition of hypogonadism used across studies varied considerably: the Study of Health in Pomerania (SHIP, 1997-2001) (Germany) [5] used the most liberal definition of hypogonadism (TT < $300 \mathrm{ng} / \mathrm{dL}$, no symptom criteria) and reported the highest prevalence at $12.8 \%$; the European Male Aging Study (EMAS, 2003-2005) [4] used the most stringent definition of symptomatic hypogonadism
(TT $<317 \mathrm{ng} / \mathrm{dL}$ plus 3 specific symptoms of decreased frequency of morning erections, sexual thoughts, and erectile dysfunction) and reported the lowest prevalence at $2.1 \%$. The Boston Area Community Health Survey (BACH, 20022005) [3] and the Massachusetts Male Aging Study (MMAS, 1987-1989) [2], both USA based, used similar operational definitions of symptomatic hypogonadism (TT cut-offs of $<300 \mathrm{ng} / \mathrm{dL}$ plus $\geq 1$ specific symptom or $\geq 2$ nonspecific symptoms $(\mathrm{BACH})$ and either TT $<200 \mathrm{ng} / \mathrm{dL}$ plus $\geq 3$ symptoms or TT $200-400 \mathrm{ng} / \mathrm{dL}$ plus FT $<8.91 \mathrm{ng} / \mathrm{dL}$ plus $\geq 3$ symptoms (see Table 2)) with reported prevalence of $5.6 \%(\mathrm{BACH})$ and $6.0 \%$ (MMAS), respectively.

3.2.2. Community-Based Studies. The prevalence of hypogonadism among the 3 community-based studies ranged from 9.5\% to $31.2 \%$ (Table 2) [6-8]. All studies were cross-sectional and were conducted in Austria, China (Hong Kong), and Malaysia. The sample sizes ranged from 247 to $351 \mathrm{men}$, and the mean (SD) ages of men varied from 54 (NR) to $75.8(0.4)$ years. The operational definition of hypogonadism varied considerably, with TT cut-offs ranging from $<200$ to $<400$, with and without low FT, and with and without symptoms. The highest prevalence $(31.2 \%)$ was reported in a study of men with a mean age of 75.8 years [6].

3.2.3. Primary Care/Screening-Based Studies. Seven crosssectional studies, conducted in Brazil, Chile, India, Italy, Taiwan, and USA, examined the prevalence of hypogonadism in a primary care or screening-based settings [9-15] (Table 2). Prevalence was higher than that observed in the general population surveys, ranging from $12 \%[10,12]$ to $38.7 \%$ [13], with the majority around $20 \%[11,12,14,15]$. The sample sizes 
TABLE 2: Prevalence of hypogonadism in population-, community-, and primary care or screening-based populations.

\begin{tabular}{|c|c|c|c|c|c|c|}
\hline $\begin{array}{l}\text { Author, year, and study } \\
\text { name }\end{array}$ & $\begin{array}{l}\text { Country and years of } \\
\text { data collected }\end{array}$ & Study design & $N$ & $\begin{array}{l}\text { Age, mean (SD), } \\
\text { and range }\end{array}$ & $\begin{array}{l}\text { Definition of } \\
\text { hypogonadism used }\end{array}$ & $\begin{array}{c}\text { Reported } \\
\text { prevalence (\%) }\end{array}$ \\
\hline \multicolumn{7}{|c|}{ Population-based studies } \\
\hline $\begin{array}{l}\text { Haring et al., } 2010 \text { [5] } \\
\text { (SHIP) }\end{array}$ & Germany, 1997-2001 & Population-based & 1490 & $\begin{array}{c}50.65(15.41) \\
\text { NR }\end{array}$ & $\mathrm{TT}<300 \mathrm{ng} / \mathrm{dL}$ & 12.8 \\
\hline $\begin{array}{l}\text { Araujo et al., } 2007 \text { [3] } \\
(\mathrm{BACH})\end{array}$ & USA, 2002-2005 & Population-based & 1475 & 47.3 (12.5), NR & $\begin{array}{l}\mathrm{TT}<300 \mathrm{ng} / \mathrm{dL}+ \\
\mathrm{FT}<5 \mathrm{ng} / \mathrm{dL}+ \\
\geq 1 \text { specific symptom or } \geq \\
2 \text { nonspecific symptoms }\end{array}$ & 5.6 \\
\hline $\begin{array}{l}\text { Araujo et al., } 2004 \text { [2] } \\
\text { (MMAS) }\end{array}$ & USA, 1987-1997 & Population-based & 1691 & $\begin{array}{l}\text { NR (NR), } \\
40-70^{* *}\end{array}$ & $\begin{array}{l}\mathrm{TT}<200 \mathrm{ng} / \mathrm{dL}+ \\
\geq 3 \text { symptoms } \\
\text { OR: } \\
\text { TT } 200-400 \mathrm{ng} / \mathrm{dL}+ \\
\text { FT }<8.91 \mathrm{ng} / \mathrm{dL}+ \\
\geq 3 \text { symptoms }^{\dagger \dagger}\end{array}$ & 6 \\
\hline $\begin{array}{l}\text { Tajar et al., } 2012 \text { [4] } \\
\text { (EMAS) }\end{array}$ & $\begin{array}{l}8 \text { European countries, } \\
2003-2005\end{array}$ & Population-based $\mathrm{d}^{\dagger \dagger}$ & 2966 & $\begin{array}{c}59.15(10.82) \\
40-79\end{array}$ & $\begin{array}{l}\mathrm{TT}<317 \mathrm{ng} / \mathrm{dL}+ \\
3 \text { specific symptoms* }\end{array}$ & 2.1 \\
\hline
\end{tabular}
Community-based studies

Ponholzer et al., 2010

[6]

Austria, 2000-2002 Community-based $247 \quad 75.8(0.4), 75-78 \quad \mathrm{TT}<350 \mathrm{ng} / \mathrm{dL}$

Khoo et al., 2008 [7]

Malaysia, NR

Community-based

$35158(7), 50-93 \quad \mathrm{TT}<317 \mathrm{ng} / \mathrm{dL}$

$\mathrm{TT}<317 \mathrm{ng} / \mathrm{dL}+$ positive symptoms on

ADAM

$\mathrm{TT}<200 \mathrm{ng} / \mathrm{dL}+$ $\geq 3$ of 7 symptoms

Wong et al., 2006 [8]

China (Hong Kong),

Community-based $252 \quad 54$ (NR), 45-64

OR: 2003-2004

TT 200-400 ng/dL +

$\mathrm{FT}<0.3093 \mathrm{nmoL} / \mathrm{L}+$ $\geq 3$ of 7 symptoms

\begin{tabular}{|c|c|c|c|c|c|c|}
\hline \multicolumn{7}{|c|}{ Primary care and screening studies } \\
\hline $\begin{array}{l}\text { Mulligan et al., } 2006 \\
\text { [13] (HIM) }\end{array}$ & USA, 2003-2004 & Primary care patients & 2162 & $\begin{array}{c}60.5(10.33) \\
45-96\end{array}$ & $\mathrm{TT}<300 \mathrm{ng} / \mathrm{dL}$ & 38.7 \\
\hline $\begin{array}{l}\text { Schneider et al., } 2009 \\
\text { (DETECT) [15] }\end{array}$ & Germany, 2003 & Primary care patients & 2719 & 58.7 (13.4), NR & $\mathrm{TT}<300 \mathrm{ng} / \mathrm{dL}$ & 19.3 \\
\hline $\begin{array}{l}\text { Nardozza et al., } 2011 \\
{[14]}\end{array}$ & Brazil, 2009 & Health screening & 1623 & 57 (NR), 24-87 & $\mathrm{TT}<300 \mathrm{ng} / \mathrm{dL}$ & 19.8 \\
\hline \multirow[t]{2}{*}{ Goel et al., 2009 [11] } & \multirow[t]{2}{*}{ India, 2006} & \multirow[t]{2}{*}{ Health Screening ${ }^{\dagger \dagger \dagger}$} & \multirow[t]{2}{*}{157} & \multirow[t]{2}{*}{$\begin{array}{l}53.1(\mathrm{NR}) \\
\quad 40-60\end{array}$} & $\mathrm{TT}<300 \mathrm{ng} / \mathrm{dL}$ & 24.2 \\
\hline & & & & & $\begin{array}{l}\mathrm{TT}<300 \mathrm{ng} / \mathrm{dL}+ \\
\text { Symptoms }\end{array}$ & 20.4 \\
\hline \multirow[t]{2}{*}{ Liu et al., 2009 [12] } & \multirow[t]{2}{*}{ Taiwan, 2007-2008 } & \multirow[t]{2}{*}{ Health screening } & \multirow[t]{2}{*}{734} & \multirow[t]{2}{*}{$57.4(6.7), 43-87$} & $\mathrm{TT}<300 \mathrm{ng} / \mathrm{dL}$ & 24.1 \\
\hline & & & & & $\begin{array}{l}\mathrm{TT}<300 \mathrm{ng} / \mathrm{dL}+ \\
\mathrm{FT}<5 \mathrm{ng} / \mathrm{dL}+\text { symptoms }\end{array}$ & 12 \\
\hline Blümel et al., 2009 [9] & Chile, NR & Primary care patients & 96 & $\begin{array}{l}55.1(12.0) \\
\quad 40-85\end{array}$ & $\begin{array}{l}\mathrm{BT}<198.4 \mathrm{ng} / \mathrm{dL}+ \\
\text { symptoms*** }\end{array}$ & 28.1 \\
\hline Di Luigi et al., 2010 [10] & Italy & Health screening & 183 & $61.9(7.5), 50-75$ & $\mathrm{TT}<230 \mathrm{ng} / \mathrm{dL}$ & 12 \\
\hline \multicolumn{7}{|c|}{$\begin{array}{l}\text { TT: } 200 \mathrm{ng} / \mathrm{dL}=6.94 \mathrm{nmol} / \mathrm{L} ; 230 \mathrm{ng} / \mathrm{dL}=8 \mathrm{nmol} / \mathrm{L} ; 300 \mathrm{ng} / \mathrm{dL}=10.4 \mathrm{nmol} / \mathrm{L} ; 317 \mathrm{ng} / \mathrm{dL}=11 \mathrm{nmol} / \mathrm{L} ; 350 \mathrm{ng} / \mathrm{dL}=12 \mathrm{nmol} / \mathrm{L} ; \mathrm{FT}: 5 \mathrm{ng} / \mathrm{dL}=0.17 \mathrm{nmol} / \mathrm{L} \text {; } \\
8.9 \mathrm{ng} / \mathrm{dL}=0.3092 \mathrm{nmoL} / \mathrm{L} \text {. } \\
\text { ADAM: androgen deficiency in the aging male; BACH: Boston Area Community Health; BT: bioavailable testosterone; cFT: calculated free testosterone; } \\
\text { DETECT: diabetes cardiovascular risk-evaluation: targets and essential data for commitment of treatment; EMAS: European Male Aging Study; HIM: } \\
\text { hypogonadism in males; MMAS: Massachusetts Male Aging Study; } \mathrm{NR}=\text { not reported; SHIP = Study of Health in Pomerania; TT = total testosterone. } \\
\text { * Decreased frequency of morning erections, sexual thoughts, and erectile dysfunction. } \\
\text { ** Participants were equally divided between men in their } 40 \mathrm{~s}, 50 \mathrm{~s} \text {, and } 60 \mathrm{~s} \text { with a similar mean age to the BACH data. } \\
{ }^{* * *} \text { Positive answer to items } 1 \text { or } 7 \text {, or any } 3 \text { other questions on the ADAM questionnaire. }\end{array}$} \\
\hline \multicolumn{7}{|c|}{$\begin{array}{l}{ }^{\dagger} \text { Specific symptoms include low libido, erective dysfunction, or osteoporosis; the nonspecific symptoms include sleep disturbance, depressed mood, lethargy, } \\
\text { or low physical performance. }\end{array}$} \\
\hline
\end{tabular}


ranged from 96 to 2,719 men and the mean (SD) age of the participants in the studies ranged from 53.1 (NR) to 61.9 (7.5) years. Six of the studies used low TT to define hypogonadism [10-15] and one study used low bioavailable $\mathrm{T}$ and symptoms [9]. Using TT $<300 \mathrm{ng} / \mathrm{dL}$ as a marker, prevalence was $19.3 \%$ [15], 19.8\% [14], 24.1\% [12], 24.2\% [11], and 38.7\% [13]. One study used a stricter TT threshold (TT $<230 \mathrm{ng} / \mathrm{dL})$ with a lower resulting prevalence of $12 \%$ [10]. Adding symptoms as a criterion, the prevalence decreased from $24.1 \%$ to $12 \%$ [12] and from $24.2 \%$ to $20.4 \%$ [11].

3.2.4. Clinical Condition-Based Studies. A total of 78 studies were identified and assessed prevalence of hypogonadism among patients with specific medical conditions (Table 3 ). The prevalence varied considerably by medical condition. Hypogonadism occurred commonly among patients with type 2 diabetes mellitus (T2DM). Thirteen studies conducted in the UK, Australia, India, Italy, and USA showed the prevalence of hypogonadism in patients with T2DM ranging from $4.4 \%$ to above $45 \%[23,25,29,36,40,45,47,48,50,56$, $61,71,87]$ varying based on the definition of hypogonadism used: prevalence ranged from $4.4 \%$ to $36 \%$ using TT $<8 \mathrm{mmol}$ $[23,29,45,50,56,61,71]$, from 24.5 to $43 \%$ using TT $<10.4 \mathrm{mmol}[36,40,47,48]$, and from $34.7 \%$ to $45 \%$ using TT $<12 \mathrm{mmol}[25,87]$. Hypogonadism was also common among obese patients with prevalence ranging from $15 \%$ to $78.8 \%$ $[22,54,74]$ and among patients with MetS, ranging from 30 to $35 \%[27,62,83]$. When combined with obesity or metabolic syndrome (MetS), prevalence estimates among patients with T2DM were higher (51\% [39] and 43\% [70] for patients with T2DM and obesity and MetS, resp.).

Prevalence of hypogonadism in patients with cardiac disease $[44,55,65,67,84,94,95]$, erectile dysfunction $[24,26$, $32-35,37,49,52,59,66,85,88]$, HIV [31, 38, 58, 78, 92, 93, 96], respiratory disease $[51,57,64]$, depression $[53,68]$, cancer $[43,46,63,75,76,79,80,82,91]$, rheumatoid arthritis [89], osteoporosis [30, 42], chronic kidney disease [21, 28, 60, 97], sickle cell disease [86], myopathies [20, 41, 52, 73, 90], urological diseases [77, 81], Peyronie's disease [69], and Alzheimer's and Parkinson's disease [72] is detailed in Table 3.

3.2.5. Prevalence of Hypogonadism by Age. A total of 10 studies, conducted in Chile, China, Europe, Italy, and USA, examined changes in prevalence of hypogonadism with age (Table 4) [2, 3, 8, 9, 12, 13, 16-19]. Sample sizes ranged from 96 to 2,162 and mean (SD) ages ranged from 47.3 (12.5) to 61.9 (7.5) years. Five of the studies were population-based[2, 3, 1719], one was community-based [8], and 4 were primary care/screening-based $[9,12,13,16]$; one was longitudinal [17] and 9 were cross-sectional $[2,3,8,9,12,13,16,18,19]$.

The prevalence of hypogonadism increased with each increasing age category $[2,3,8,9,12,13,16-19]$, with the rare exception within certain age categories $[3,8]$ (Table 4 ). Three of the studies reported significant $P$ values for trend $[2,9,12]$, 3 reported significant differences between older and younger age categories [3, 9, 18], 2 studies did not conduct statistical analysis for differences in prevalence by age $[17,19]$, and one study was nonsignificant [8]. One cross-sectional study [13] reported a $17 \%(95 \% \mathrm{CI}, 1.08-1.27)$ increase in risk of hypogonadism, for every 10 -year increase in age. Prevalence ranged from approximately $0.1 \%$ to $16.5 \%$ among men aged $40-49$, from $0.6 \%$ to $31.8 \%$ among men aged $50-59$, from $3.2 \%$ to $30.1 \%$ among men aged $60-69$, up to $66.7 \%$ among men of age $\geq 70$ years $[2,3,12,17-19]$, and $49 \%$ in the study that assessed the 80+ age category [17] (not all studies used the same age cut-offs).

3.3. Incidence of Hypogonadism. In two population-based studies (SHIP [5] and BACH [2]), in which incidence was examined, the reported incidence was 12.3 cases per 1,000 person-years in the US and 11.7 cases per 1000 person-years in Germany.

3.4. Treatment Rates for Hypogonadism. The proportion of patients receiving treatment was defined as the percentage of men with hypogonadism who were receiving testosterone therapy. Only 4 studies were identified and reported treatment proportions: one population-based [98], one primary care-based [13], and 2 clinical condition-based studies [38, 79]. The population-based (BACH) and clinical-based studies (HIM) reported that $10 \%$ [98] and $12 \%$ [13] of patients were receiving treatment for hypogonadism, respectively. Among the clinical condition-based studies, $12.1 \%$ of 144 childhood cancer survivors [79] and 38\% of 296 men with HIV were on treatment for hypogonadism [38].

\section{Discussion}

In this systematic review, we identified 97 studies that met our inclusion/exclusion criteria for assessing the epidemiology of hypogonadism. The prevalence of hypogonadism was high and varied according to the operational definition of hypogonadism used and the population studied. The prevalence of hypogonadism in the population-based, community-based, and primary care- or screening-based studies ranged from 2.1 to $12.8 \%$ [9-15], from 9.5 to $31.2 \%$ [9-18], and from 12 to $38.7 \%$ [9-15], respectively. The prevalence was higher among clinical-based populations with specific conditions. For example, among patients with T2DM, obesity, and MetS, the prevalence ranged from 4.4 to $>50 \%[23,25,29,36,40$, $45,47,48,50,56,61,71,87]$, from 15 to $78.8 \%[22,54,74]$, and from 30 to $35 \%[27,62,83]$, respectively.

In most instances, studies showed a linear relationship between prevalence and the degree of liberalness in the operational definition of hypogonadism $[4,5,7,12]$. For instance, Haring et al. [5] reported prevalence estimates below TT levels of $8.0,8.7,10.4$, and $12.0 \mathrm{nmol} / \mathrm{L}$, which were $3.4 \%, 4.5 \%$, $12.8 \%$, and $21.6 \%$. The added criteria of symptoms typically ensued a lower prevalence due to increased stringency of diagnosis. Among the population- and community-based studies, those that used a more stringent definition of hypogonadism, which included symptoms and low T, reported the lowest prevalence, ranging from $2.1 \%$ to $9.52 \%[2-4,7,8]$, while those using a more liberal definition of hypogonadism (low $\mathrm{T}$ only) reported higher prevalence, ranging from $19.1 \%$ to $31.2 \%$ [5-7]. The association between definition of 
TABLE 3: Prevalence of hypogonadism among patients with specific conditions.

\begin{tabular}{|c|c|c|c|c|c|}
\hline $\begin{array}{l}\text { Author, year, and } \\
\text { study name }\end{array}$ & $\begin{array}{l}\text { Country and years } \\
\text { of data collected }\end{array}$ & Study population & $N$ & $\begin{array}{l}\text { Definition of } \\
\text { hypogonadism used }\end{array}$ & $\begin{array}{c}\text { Prevalence (\%) } \\
\text { among patients with } \\
\text { disease of interest }\end{array}$ \\
\hline \multicolumn{6}{|c|}{ Type 2 diabetes mellitus (T2DM) } \\
\hline $\begin{array}{l}\text { Kapoor et al., } 2007 \\
\text { [56] }\end{array}$ & UK/not specified & $\begin{array}{c}\text { Primary care } \\
\text { retinopathy } \\
\text { Screening service } \\
\text { and medical clinic }\end{array}$ & 355 men with T2DM & $\begin{array}{l}\mathrm{TT}<8 \mathrm{nmol} / \mathrm{L} \\
\mathrm{TT} 8-12 \mathrm{nmol} / \mathrm{L}\end{array}$ & $\begin{array}{l}17 \\
25\end{array}$ \\
\hline $\begin{array}{l}\text { Biswas et al., } 2012 \\
{[25]}\end{array}$ & UK/not specified & Medical clinic & $\begin{array}{c}329 \\
\text { T2DM }(n=115) \\
\text { T1DM }(n=93) \\
\text { controls }(n=12)\end{array}$ & $\mathrm{TT}<11.9 \mathrm{nM}$ & 45 \\
\hline $\begin{array}{l}\text { Chen et al., } 2006 \\
{[29]}\end{array}$ & $\begin{array}{c}\text { Australia/NZ/1992 } \\
\text { to } 2000\end{array}$ & Population-based & $\begin{array}{c}195 \\
\text { T2DM }(n=35) \\
\text { Non-T2DM } \\
(n=160)\end{array}$ & $\mathrm{TT}<8.0 \mathrm{nmol} / \mathrm{L}$ & 14.3 \\
\hline $\begin{array}{l}\text { Corona et al., } 2006 \\
{[36]}\end{array}$ & Italy/not specified & Medical clinic & $\begin{array}{c}1246 \\
\text { T2DM }(n=199)\end{array}$ & $\mathrm{TT}<10.4 \mathrm{nmol} / \mathrm{L}$ & 24.5 \\
\hline $\begin{array}{l}\text { Anderson et al., } 2012 \\
\text { [23] }\end{array}$ & $\begin{array}{c}\text { UK/January } \\
\text { 2008-June } 2009\end{array}$ & Medical clinic & $\begin{array}{c}387 \\
\operatorname{T} 2 \mathrm{DM}(n=353) \\
\operatorname{T1DM}(n=23)\end{array}$ & $\begin{array}{l}\mathrm{TT}<8.0 \mathrm{nmol} / \mathrm{L} \\
\mathrm{TT} 8-11.99 \mathrm{nmol} / \mathrm{L}\end{array}$ & $\begin{array}{c}4.4 \\
32.1\end{array}$ \\
\hline $\begin{array}{l}\text { Talukder et al., } 2010 \\
\text { [87] }\end{array}$ & $\begin{array}{c}\text { India/May } \\
\text { 2008-April } 2009\end{array}$ & Medical clinic & $\begin{array}{c}420 \\
\operatorname{T2DM}(n=170) \\
\text { non-T2DM }(n=40)\end{array}$ & $\begin{array}{l}\text { Symptoms/signs of } \\
\text { hypogonadism and } \\
\mathrm{TT} \leq 12 \mathrm{nmol} / \mathrm{L}\end{array}$ & 34.7 \\
\hline $\begin{array}{l}\text { Dhindsa et al., } 2004 \\
\text { [40] }\end{array}$ & US/not specified & Medical clinic & $\begin{array}{c}103 \\
\text { men with T2DM }\end{array}$ & $\mathrm{TT}<300 \mathrm{ng} / \mathrm{dL}$ & 33 \\
\hline $\begin{array}{l}\text { Grossmann et al., } \\
2008 \text { [48] }\end{array}$ & $\begin{array}{l}\text { Australia/not } \\
\text { specified }\end{array}$ & Medical clinic & $\begin{array}{c}649 \\
\text { T2DM }(n=574) \\
\operatorname{T1DM}(n=69)\end{array}$ & $\mathrm{TT}<10 \mathrm{nmol} / \mathrm{L}$ & 43 \\
\hline $\begin{array}{l}\text { Grossmann et al., } \\
2009 \text { [47] }\end{array}$ & Australia/2005 & Medical clinic & $\begin{array}{l}464 \text { men with } \\
\text { T2DM }\end{array}$ & $\mathrm{TT}<10 \mathrm{nmol} / \mathrm{L}$ & 43 \\
\hline $\begin{array}{l}\text { La Vignera et al., } \\
2009 \text { [61] }\end{array}$ & Italy/not specified & Medical clinic & 110 men with T2DM & $\mathrm{TT}<231 \mathrm{ng} / \mathrm{mL}$ & 10 \\
\hline $\begin{array}{l}\text { Ogbera et al., } 2011 \\
\text { [71] }\end{array}$ & $\begin{array}{l}\text { Nigeria/December } \\
2009 \text { to May } 2010\end{array}$ & Medical clinic & 203 men with T2DM & $\mathrm{TT}<8 \mathrm{nmol} / \mathrm{L}$ & 36 \\
\hline $\begin{array}{l}\text { Hackett et al., } 2009 \\
\text { [50] }\end{array}$ & $\begin{array}{l}\text { UK/September } 2007 \\
\text { and May } 2008\end{array}$ & Medical clinic & 488 men with T2DM & $\begin{array}{l}\mathrm{TT}<8 \mathrm{nmol} / \mathrm{LTT} \\
8-12 \mathrm{nmol} / \mathrm{L}\end{array}$ & $\begin{array}{l}15.5 \\
33.8\end{array}$ \\
\hline $\begin{array}{l}\text { Ganesh et al., } 2009 \\
\text { [45] }\end{array}$ & India/not specified & Medical clinic & 100 men with T2DM & $\begin{array}{l}\mathrm{cFT}<64.8 \mathrm{pg} / \mathrm{mL} \\
(0.225 \mathrm{nmol} / \mathrm{L})\end{array}$ & 15 \\
\hline \multicolumn{6}{|c|}{ tabolic syndrome (MetS) } \\
\hline \multirow{3}{*}{ Ogbera $2011[70]$} & \multirow{3}{*}{$\begin{array}{l}\text { Nigeria/December } \\
2009 \text { to May } 2010\end{array}$} & \multirow{3}{*}{ Medical clinic } & \multirow{3}{*}{$\begin{array}{l}203 \text { men with T2DM } \\
+ \text { MetS }(n=90)\end{array}$} & $\begin{array}{l}\mathrm{T} 2 \mathrm{DM}+\mathrm{TT}< \\
12 \mathrm{nmol} / \mathrm{L}\end{array}$ & 36 \\
\hline & & & & $\mathrm{T} 2 \mathrm{DM}+\mathrm{MetS}$ & 43 \\
\hline & & & & $\begin{array}{l}\text { T2DM + MetS + low } \\
\text { TT }\end{array}$ & 47 \\
\hline \multirow{3}{*}{$\begin{array}{l}\text { Dhindsa et al., } 2010 \\
\text { [39] }\end{array}$} & \multirow{3}{*}{ US/not specified } & \multirow{3}{*}{ Medical clinic } & 1849 & $\begin{array}{l}\mathrm{FT}<300 \mathrm{ng} / \mathrm{dL} \text { or } \\
10.4 \mathrm{nmol} / \mathrm{L}:\end{array}$ & \\
\hline & & & $\begin{array}{l}\text { Obesity + T2DM } \\
\quad(n=398)\end{array}$ & Obesity and T2DM & 51 \\
\hline & & & Obesity $(n=398)$ & Obese & 31 \\
\hline \multicolumn{6}{|c|}{ Obesity } \\
\hline $\begin{array}{l}\text { Hofstra et al., } 2008 \\
{[54]}\end{array}$ & $\begin{array}{l}\text { The Netherlands/not } \\
\text { specified }\end{array}$ & Medical clinic & 160 obese males & $\mathrm{TT}<11 \mathrm{nmol} / \mathrm{L}$ & 57.7 \\
\hline
\end{tabular}


TABLe 3: Continued.

\begin{tabular}{|c|c|c|c|c|c|}
\hline $\begin{array}{l}\text { Author, year, and } \\
\text { study name }\end{array}$ & $\begin{array}{l}\text { Country and years } \\
\text { of data collected }\end{array}$ & Study population & $N$ & $\begin{array}{l}\text { Definition of } \\
\text { hypogonadism used }\end{array}$ & $\begin{array}{c}\text { Prevalence (\%) } \\
\text { among patients with } \\
\text { disease of interest }\end{array}$ \\
\hline \multirow{4}{*}{$\begin{array}{l}\text { Pellitero et al., } 2012 \\
\text { [74] }\end{array}$} & \multirow{4}{*}{ Spain/not specified } & \multirow{4}{*}{$\begin{array}{l}\text { Clinical research } \\
\text { center }\end{array}$} & \multirow{4}{*}{33 obese males } & $\mathrm{TT}<300 \mathrm{ng} / \mathrm{dL}$ & 78.8 \\
\hline & & & & $\begin{array}{l}\mathrm{TT}<200 \mathrm{ng} / \mathrm{dL}+ \\
\mathrm{FT}<65 \mathrm{pg} / \mathrm{mL}\end{array}$ & 100 \\
\hline & & & & $\begin{array}{l}\mathrm{TT}<300 \text { but } \\
>200 \mathrm{ng} / \mathrm{dL}\end{array}$ & 23.1 \\
\hline & & & & $\begin{array}{l}\text { FT low and TT > } \\
300 \mathrm{ng} / \mathrm{dL}\end{array}$ & 3.85 \\
\hline \multirow{3}{*}{$\begin{array}{l}\text { Allan et al., } 2006 \\
{[22]}\end{array}$} & \multirow{3}{*}{$\begin{array}{l}\text { Australia/May } \\
\text { 2001-February } 2003\end{array}$} & \multirow{3}{*}{ Community-based } & 223 & $\begin{array}{l}\mathrm{TT}<8 \mathrm{nmol} / \mathrm{L} \text { plus } \\
\text { symptoms }\end{array}$ & \\
\hline & & & Obese $(n=99)$ & Obese & 15 \\
\hline & & & Nonobese $(n=124)$ & Nonobese & 3 \\
\hline \multicolumn{6}{|c|}{ MetS } \\
\hline $\begin{array}{l}\text { Laaksonen et al., } \\
2005 \text { [62] }\end{array}$ & Finland/1987-2001 & Population-based & 854 men with MetS & $\mathrm{TT}<11 \mathrm{nmol} / \mathrm{L}$ & 10.7 \\
\hline Singh et al., 2011 [83] & India/not specified & Medical clinic & 95 men with MetS & $\mathrm{FT}<0.225 \mathrm{nmol} / \mathrm{L}$ & 30.2 \\
\hline $\begin{array}{l}\text { Caldas et al., } 2009 \\
{[27]}\end{array}$ & $\begin{array}{l}\text { Brazil/January } 2003 \\
\text { to March } 2007 \\
\end{array}$ & Medical clinic & 80 men with MetS & $\mathrm{TT}<300 \mathrm{ng} / \mathrm{dL}$ & 30.0 \\
\hline \multicolumn{6}{|c|}{ Cardiac disease } \\
\hline $\begin{array}{l}\text { Jankowska et al., } \\
2006[55]\end{array}$ & $\begin{array}{l}\text { Poland/October } \\
2001 \text { to November } \\
2002\end{array}$ & Medical clinic & $\begin{array}{c}574 \\
\text { CHF }(n=208) \\
\text { health controls } \\
(n=366)\end{array}$ & $\begin{array}{l}\text { Serum TT and FT } \\
\text { levels at or below the } \\
\text { 10th percentile of } \\
\text { healthy peers }\end{array}$ & 13-39 (by age) \\
\hline $\begin{array}{l}\text { Wang et al., } 2010 \\
{[94]}\end{array}$ & $\begin{array}{c}\text { China/March 1, } \\
\text { 2005, to May 31, } \\
2007\end{array}$ & Medical clinic & 175 men with CHF & $\begin{array}{l}\text { Serum levels at or } \\
\text { below the 10th } \\
\text { percentile of healthy } \\
\text { peers }\end{array}$ & 21.7 \\
\hline Serra et al., 2012 [84] & Italy/not specified & Medical clinic & $\begin{array}{l}52 \text { male heart } \\
\text { transplantation } \\
\text { recipients }\end{array}$ & $\mathrm{TT}<10.4 \mathrm{nmol} / \mathrm{L}$ & 34.6 \\
\hline $\begin{array}{l}\text { Florvaag et al., } 2012 \\
\text { [44] }\end{array}$ & $\begin{array}{l}\text { Germany/not } \\
\text { specified }\end{array}$ & Medical clinic & $\begin{array}{l}137 \text { male heart } \\
\text { failure patients }\end{array}$ & $\begin{array}{l}\text { Free testosterone } \\
\text { (age-related cut-off } \\
\text { values) }\end{array}$ & 39 \\
\hline $\begin{array}{l}\text { Malkin et al., } 2010 \\
\text { [67] }\end{array}$ & $\begin{array}{l}\text { UK/June } 2000 \text { and } \\
\text { June } 2002\end{array}$ & Medical clinic & $\begin{array}{l}930 \text { men with } \\
\text { coronary disease }\end{array}$ & $\mathrm{TT}<8.1 \mathrm{nmol} / \mathrm{L}$ & 16.9 \\
\hline Wehr et al., 2010 [95] & $\begin{array}{c}\text { Germany/1997 to } \\
2000\end{array}$ & Medical clinic & $\begin{array}{l}2299 \text { men who were } \\
\text { routinely referred } \\
\text { for coronary } \\
\text { angiography }\end{array}$ & $\mathrm{T}<11.3 \mathrm{nmol} / \mathrm{L}$ & 18 \\
\hline $\begin{array}{l}\text { Lerchbaum et al., } \\
2012 \text { [65] }\end{array}$ & Germany/1997-2000 & Medical clinic & $\begin{array}{l}2069 \text { men with } \\
\text { coronary disease }\end{array}$ & $\mathrm{TT}<11.3 \mathrm{nM}$ & 18.4 \\
\hline \multicolumn{6}{|c|}{ Erectile dysfunction (ED) } \\
\hline $\begin{array}{l}\text { Köhler et al., } 2008 \\
\text { [59] }\end{array}$ & US/1987 to 2002 & Chart review & $\begin{array}{l}2794 \text { men presenting } \\
\text { for ED evaluation }\end{array}$ & $\mathrm{TT}<300 \mathrm{ng} / \mathrm{dL}$ & 23 \\
\hline $\begin{array}{l}\text { Bunch et al., } 2002 \\
{[26]}\end{array}$ & US/1996 to 1999 & Chart review & 501 men with ED & $\mathrm{TT}<300 \mathrm{ng} / \mathrm{dL}$ & 44 \\
\hline $\begin{array}{l}\text { Corona et al., } 2009 \\
\text { [33] }\end{array}$ & Italy/not specified & Chart review & 2302 men with ED & $\begin{array}{l}\mathrm{TT}<12 \mathrm{nmol} / \mathrm{L}+ \\
\text { symptoms }\end{array}$ & 28.1 \\
\hline Arafa et al., 2012 [24] & Egypt/not specified & Medical clinic & $\begin{array}{c}212 \text { men } \mathrm{T} 2 \mathrm{DM}+ \\
\text { ED }\end{array}$ & $\begin{array}{l}\mathrm{TT}<300 \mathrm{ng} / \mathrm{dL}+ \\
\text { symptoms }\end{array}$ & 41 \\
\hline Guay et al., 2010 [49] & $\begin{array}{c}\text { US/July } 1995 \text { to July } \\
1997\end{array}$ & Chart review & 990 with men ED & $\begin{array}{l}10.4 \mathrm{nmol} / \mathrm{L}+ \\
\text { symptoms }\end{array}$ & 36.3 \\
\hline
\end{tabular}


TABle 3: Continued.

\begin{tabular}{|c|c|c|c|c|c|}
\hline $\begin{array}{l}\text { Author, year, and } \\
\text { study name }\end{array}$ & $\begin{array}{l}\text { Country and years } \\
\text { of data collected }\end{array}$ & Study population & $N$ & $\begin{array}{l}\text { Definition of } \\
\text { hypogonadism used }\end{array}$ & $\begin{array}{c}\text { Prevalence (\%) } \\
\text { among patients with } \\
\text { disease of interest }\end{array}$ \\
\hline $\begin{array}{l}\text { Makhlouf et al., } \\
2008[66]\end{array}$ & $\begin{array}{l}\text { US/July } 2001 \text { to June } \\
2003\end{array}$ & Medical clinic & 157 men with ED & $\mathrm{TT}<300 \mathrm{mg} / \mathrm{dL}$ & 36 \\
\hline $\begin{array}{l}\text { Corona et al., } 2007 \\
\text { [35] }\end{array}$ & Italy/not specified & Medical clinic & 1134 men with ED & $\mathrm{TT}<10.4 \mathrm{nmol} / \mathrm{L}$ & 16.9 \\
\hline $\begin{array}{l}\text { Corona et al., } 2010 \\
\text { [37] }\end{array}$ & Italy/2000 to 2007 & Medical clinic & 1687 men with ED & $\begin{array}{l}\text { TT } 230-300 \mathrm{ng} / \mathrm{dL} \\
\text { no symptoms }\end{array}$ & 13.3 \\
\hline $\begin{array}{l}\text { Corona et al., } 2009 \\
\text { [34] }\end{array}$ & $\begin{array}{l}\text { Italy/January } 2001 \text { to } \\
\text { April } 2008\end{array}$ & Medical clinic & 1093 men with ED & & \\
\hline $\begin{array}{l}\text { Somani et al., } 2010 \\
\text { [85] }\end{array}$ & $\begin{array}{c}\text { UK/March } \\
\text { 2007-August } 2008\end{array}$ & Medical clinic & 124 men with ED & $\begin{array}{l}\text { Low TT (not further } \\
\text { defined) }\end{array}$ & 23.4 \\
\hline $\begin{array}{l}\text { Corona et al., } 2010 \\
\text { [32] }\end{array}$ & $\begin{array}{l}\text { Italy/January } \\
\text { 2001-April } 2009\end{array}$ & Medical chart review & $\begin{array}{l}3712 \text { men with } \\
\text { sexual dysfunction }\end{array}$ & $\begin{array}{l}\mathrm{TT}<8 \mathrm{nmol} / \mathrm{L}+ \\
\text { symptoms }\end{array}$ & 7.5 \\
\hline Tan et al., 2011 [88] & $\begin{array}{c}\text { Multiethnic } \\
\text { Asia/Pacific/2003 }\end{array}$ & Medical chart review & $\begin{array}{c}1046 \\
\operatorname{ED}(n=194)\end{array}$ & $\mathrm{TT}<10.4 \mathrm{nmol} / \mathrm{L}$ & 20.4 \\
\hline $\begin{array}{l}\text { Hamidi et al., } 2012 \\
\text { [52] }\end{array}$ & $\begin{array}{c}\text { Iran/November } \\
2009 \text { to August } 2010\end{array}$ & Medical clinic & 241 men with ED & $\mathrm{FT}<5 \mathrm{ng} / \mathrm{dL}$ & 36.5 \\
\hline \multicolumn{6}{|c|}{ HIV } \\
\hline $\begin{array}{l}\text { Klein et al., } 2005 \\
{[58]}\end{array}$ & US/not specified & Community-based & $\begin{array}{c}502 \text { (had or at risk of } \\
\text { HIV) }\end{array}$ & $\mathrm{TT}<300 \mathrm{ng} / \mathrm{dL}$ & 45.6 \\
\hline $\begin{array}{l}\text { Crum-Cianflone et } \\
\text { al., } 2007 \text { [38] }\end{array}$ & $\begin{array}{l}\text { US/September } 2004 \\
\quad \text { to May } 2005\end{array}$ & HIV medical clinic & $300 \mathrm{HIV}+$ & $\mathrm{TT}<300 \mathrm{ng} / \mathrm{dL}$ & 17 \\
\hline $\begin{array}{l}\text { Wahlstrom et al., } \\
2000 \text { [93] }\end{array}$ & US/not specified & Observational study & $20 \mathrm{HIV}+$ & $\mathrm{T} \leq 410 \mathrm{ng} / \mathrm{dL}$ & 25 \\
\hline $\begin{array}{l}\text { Collazos et al., } 2009 \\
\text { [31] }\end{array}$ & Spain/not specified & Medical chart review & $188 \mathrm{HIV}+$ & $\mathrm{T}<2.6 \mathrm{ng} / \mathrm{mL}$ & 3.7 \\
\hline $\begin{array}{l}\text { Wunder et al., } 2007 \\
{[96]}\end{array}$ & $\begin{array}{l}\text { Switzerland/not } \\
\text { specified }\end{array}$ & Cohort study & $139 \mathrm{HIV}+$ & $\begin{array}{l}\text { FT below the } \\
\text { age-adjusted normal } \\
\text { limits }\end{array}$ & 70 \\
\hline $\begin{array}{l}\text { Wagner et al., } 1995 \\
\text { [92] }\end{array}$ & US/not specified & $\begin{array}{l}\text { Study setting } \\
\text { (baseline data) }\end{array}$ & $234 \mathrm{HIV}+$ & $\mathrm{TT} \leq 400 \mathrm{ng} / \mathrm{dL}$ & 38 \\
\hline $\begin{array}{l}\text { Rietschel et al., } 2000 \\
{[78]}\end{array}$ & $\begin{array}{c}\text { US/November } \\
\text { 1997-April } 1999\end{array}$ & Cohort study & $90 \mathrm{HIV}+$ & Low TT/FT & 19 \\
\hline \multicolumn{6}{|c|}{ Respiratory disease } \\
\hline $\begin{array}{l}\text { Laghi et al., } 2005 \\
{[64]}\end{array}$ & US/not specified & Cohort study & 101 men with COPD & $\mathrm{FT}<50 \mathrm{pg} / \mathrm{mL}$ & 37.6 \\
\hline $\begin{array}{l}\text { Kirbas et al., } 2007 \\
\text { [57] }\end{array}$ & $\begin{array}{l}\text { Turkey/November } \\
2004 \text { to July } 2006\end{array}$ & Medical clinic & $\begin{array}{c}96 \text { men with } \\
\text { obstructive sleep } \\
\text { apnea }\end{array}$ & $\mathrm{TT}<2.62 \mathrm{ng} / \mathrm{mL}$ & 19.8 \\
\hline $\begin{array}{l}\text { Halabi et al., } 2011 \\
\text { [51] }\end{array}$ & US/not specified & Medical clinic & 104 men with COPD & $\begin{array}{l}\mathrm{FT}<35 \mathrm{pg} / \mathrm{mL} \text { for } \\
\text { patients }<70 \text { years; } \\
\mathrm{FT}<30 \mathrm{pg} / \mathrm{mL} \text { for } \\
\text { patients } \geq 70 \text { years }\end{array}$ & 33.6 \\
\hline \multirow{3}{*}{$\begin{array}{l}\text { Hintikka et al., } 2009 \\
\text { [53] }\end{array}$} & \multirow{3}{*}{$\begin{array}{l}\text { Finland/1998, 1999, } \\
\text { 2001, and 2005 }\end{array}$} & \multirow{3}{*}{ Population-based } & \multirow{3}{*}{$\begin{array}{l}116 \\
\text { with and without } \\
\text { psychological } \\
\text { symptoms }\end{array}$} & & \\
\hline & & & & $\begin{array}{l}\mathrm{FT}<160 \mathrm{pmol} / \mathrm{L} \\
\text { plus BDI scores: } \\
\text { +depressive } \\
\text { symptoms }\end{array}$ & 28.8 \\
\hline & & & & $\begin{array}{l}\text {-depressive } \\
\text { symptoms }\end{array}$ & 10.5 \\
\hline \multirow[t]{2}{*}{$\begin{array}{l}\text { McIntyre et al., } 2006 \\
\text { [68] }\end{array}$} & \multirow[t]{2}{*}{$\begin{array}{l}\text { Canada/not } \\
\text { specified }\end{array}$} & \multirow[t]{2}{*}{ Medical clinic } & \multirow[t]{2}{*}{$\begin{array}{l}94 \text { men with and } \\
\text { without depression }\end{array}$} & $\begin{array}{l}\mathrm{TT}<12.14 \mathrm{nmol} / \mathrm{L}: \\
\text { Men with depression }\end{array}$ & 34 \\
\hline & & & & $\begin{array}{l}\text { Men without } \\
\text { depression }\end{array}$ & 6 \\
\hline
\end{tabular}


TABle 3: Continued.

\begin{tabular}{|c|c|c|c|c|c|}
\hline $\begin{array}{l}\text { Author, year, and } \\
\text { study name }\end{array}$ & $\begin{array}{l}\text { Country and years } \\
\text { of data collected }\end{array}$ & Study population & $N$ & $\begin{array}{l}\text { Definition of } \\
\text { hypogonadism used }\end{array}$ & $\begin{array}{c}\text { Prevalence (\%) } \\
\text { among patients with } \\
\text { disease of interest }\end{array}$ \\
\hline \multicolumn{6}{|c|}{ Cancer } \\
\hline $\begin{array}{l}\text { Tromp et al., } 2011 \\
\text { [91] }\end{array}$ & $\begin{array}{l}\text { The } \\
\text { Netherlands/2008 }\end{array}$ & Medical clinics & $\begin{array}{l}565 \text { male } 5 \text {-year } \\
\text { survivors of } \\
\text { childhood cancer }\end{array}$ & $\mathrm{T}<11.0 \mathrm{nmol} / \mathrm{L}$ & 12.4 \\
\hline $\begin{array}{l}\text { Lackner et al., } 2007 \\
\text { [63] }\end{array}$ & Austria/not specified & Medical clinic & $\begin{array}{l}68 \text { men with } \\
\text { testicular cancer }\end{array}$ & $\begin{array}{l}\mathrm{T}<3.0 \mathrm{ng} / \mathrm{mL}+ \\
\text { symptoms }\end{array}$ & 33.8 \\
\hline Pühse et al., 2011 [75] & Germany/2004 & Medical clinic & $\begin{array}{l}376 \text { men with } \\
\text { testicular germ-cell } \\
\text { cancer (TGCC) }\end{array}$ & $\begin{array}{l}\text { TT } 2.84 \mathrm{ng} / \mathrm{mL} \text { or } \\
9.85 \mathrm{nmol} / \mathrm{L}\end{array}$ & $\sim 25$ \\
\hline $\begin{array}{l}\text { Fleishman et al., } \\
2010 \text { [43] }\end{array}$ & $\begin{array}{c}\text { US/July } 2007 \\
\text { through July } 2009\end{array}$ & Medical clinic & $\begin{array}{c}428 \text { male patients } \\
\text { with } \\
\text { nontestosterone- } \\
\text { related } \\
\text { cancer }\end{array}$ & $\mathrm{TT}<300 \mathrm{ng} / \mathrm{dL}$ & 48 \\
\hline $\begin{array}{l}\text { Salonia et al., } 2011 \\
{[80]}\end{array}$ & $\begin{array}{l}\text { Italy/June } 2006 \text { and } \\
\text { June } 2008\end{array}$ & Medical clinic & $\begin{array}{l}673 \text { prostate cancer } \\
\text { patients }\end{array}$ & $\mathrm{TT}<3 \mathrm{ng} / \mathrm{mL}$ & $21.4 \%$ \\
\hline $\begin{array}{l}\text { Greenfield et al., } \\
2007 \text { [46] }\end{array}$ & US/not specified & Medical clinic & 389 cancer survivors & $\mathrm{TT}<10 \mathrm{nmol} / \mathrm{L}$ & 13.6 \\
\hline \multirow{4}{*}{$\begin{array}{l}\text { Rajagopal et al., } \\
2004 \text { [76] }\end{array}$} & \multirow{4}{*}{ US/not specified } & \multirow{4}{*}{ Chart review } & \multirow{4}{*}{40 cancer survivors } & $<345 \mathrm{ng} / \mathrm{dL}$ & \\
\hline & & & & All & 65 \\
\hline & & & & On opioids & 90 \\
\hline & & & & Not on opioids & 40 \\
\hline $\begin{array}{l}\text { Schatzl et al., } 2001 \\
\text { [82] }\end{array}$ & $\begin{array}{c}\text { Austria/January } \\
\text { 1999-April } 2000\end{array}$ & Medical clinic & $\begin{array}{l}156 \text { men with } \\
\text { prostate cancer }\end{array}$ & $\mathrm{TT}<3.0 \mathrm{ng} / \mathrm{mL}$ & 33.3 \\
\hline $\begin{array}{l}\text { Romerius et al., } \\
2009 \text { [79] }\end{array}$ & Sweden/2004-2006 & Medical clinic & $\begin{array}{l}292 \text { men with } \\
\text { history of childhood } \\
\text { treatment of cancer } \\
\text { or brain tumor }\end{array}$ & $\mathrm{T}<10 \mathrm{nmol} / \mathrm{L}$ & 23 \\
\hline \multicolumn{6}{|c|}{ Rheumatoid arthritis (RA) } \\
\hline $\begin{array}{l}\text { Tengstrand et al., } \\
2002 \text { [89] }\end{array}$ & $\begin{array}{l}\text { Sweden/not } \\
\text { specified }\end{array}$ & Medical clinic & 104 patients with RA & $\begin{array}{l}<6.5 \mathrm{nmol} / \mathrm{L}- \\
8.1 \mathrm{nmol} / \mathrm{L} \\
\text { (age dependent) }\end{array}$ & 31.7 \\
\hline \multicolumn{6}{|c|}{ Osteoporosis } \\
\hline Fink et al., 2006 [42] & $\begin{array}{c}\text { US/March } 2000 \text { to } \\
\text { April } 2002\end{array}$ & Medical clinics & $\begin{array}{c}3674 \text { men with } \\
\text { osteoporosis }\end{array}$ & $\mathrm{TT}<200 \mathrm{ng} / \mathrm{dL}$ & 6.9 \\
\hline $\begin{array}{l}\text { Clapauch et al., } 2008 \\
{[30]}\end{array}$ & Brazil/January 2005 & Medical clinic & $\begin{array}{c}216 \text { men with } \\
\text { osteoporosis }\end{array}$ & $\begin{array}{l}\mathrm{cFT}<6.5 \mathrm{ng} / \mathrm{dL}+ \\
\text { symptoms }\end{array}$ & 25 \\
\hline \multicolumn{6}{|c|}{ Chronic kidney disease (CKD) } \\
\hline $\begin{array}{l}\text { Yilmaz et al., } 2011 \\
\text { [97] }\end{array}$ & $\begin{array}{l}\text { Turkey/March } 2006 \\
\text { to June } 2010\end{array}$ & Medical clinic & 239 men with CKD & $\mathrm{TT} 10 \mathrm{nmol} / \mathrm{L}$ & 33 \\
\hline $\begin{array}{l}\text { Carrero et al., } 2011 \\
{[28]}\end{array}$ & $\begin{array}{l}\text { Sweden/June } 1999 \\
\text { and October } 2007\end{array}$ & Medical clinics & 260 men with ESRD & $\mathrm{TT}<10 \mathrm{nmol} / \mathrm{L}$ & 44 \\
\hline $\begin{array}{l}\text { Kyriazis et al., } 2011 \\
{[60]}\end{array}$ & $\begin{array}{c}\text { Greece/October } \\
2005 \text { and December } \\
2006\end{array}$ & Medical clinic & $\begin{array}{l}111 \text { male } \\
\text { hemodialysis } \\
\text { patients }\end{array}$ & $\mathrm{TT}<8 \mathrm{nmol} / \mathrm{L}$ & 49 \\
\hline $\begin{array}{l}\text { Albaaj et al., } 2006 \\
{[21]}\end{array}$ & UK/not specified & Medical clinic & $\begin{array}{l}214 \text { male patients } \\
\text { with CKD or ESRD }\end{array}$ & $\mathrm{TT}<10 \mathrm{nmol} / \mathrm{L}$ & 26.2 \\
\hline \multicolumn{6}{|c|}{ Myopathies } \\
\hline $\begin{array}{l}\text { Travison et al., } 2008 \\
\text { [90] }\end{array}$ & Canada/3-year study & Medical clinic & $\begin{array}{l}59 \text { men with } \\
\text { myopathies }\end{array}$ & $10-30 \mathrm{nmol} / \mathrm{L}$ & 54 \\
\hline $\begin{array}{l}\text { Durga et al., } 2011 \\
{[41]}\end{array}$ & $\begin{array}{c}\text { US/July } 2006 \text { to } \\
\text { April } 2007\end{array}$ & Medical clinic & $\begin{array}{l}60 \text { men with spinal } \\
\text { cord injuries }\end{array}$ & $\mathrm{TT}<325 \mathrm{ng} / \mathrm{dL}$ & 43.3 \\
\hline
\end{tabular}


TABLe 3: Continued.

\begin{tabular}{|c|c|c|c|c|c|}
\hline $\begin{array}{l}\text { Author, year, and } \\
\text { study name }\end{array}$ & $\begin{array}{l}\text { Country and years } \\
\text { of data collected }\end{array}$ & Study population & $N$ & $\begin{array}{l}\text { Definition of } \\
\text { hypogonadism used }\end{array}$ & $\begin{array}{c}\text { Prevalence (\%) } \\
\text { among patients with } \\
\text { disease of interest }\end{array}$ \\
\hline $\begin{array}{l}\text { Okun et al., } 2002 \\
\text { [73] }\end{array}$ & US/not specified & Medical clinic & 68 men with PD & $\mathrm{FT}<70 \mathrm{pg} / \mathrm{mL}$ & 35 \\
\hline $\begin{array}{l}\text { Abbasi et al., } 1994 \\
{[20]}\end{array}$ & US/not specified & Medical clinic & $\begin{array}{c}102 \text { men with } \\
\text { poststroke } \\
\text { hemiplegia }\end{array}$ & $\begin{array}{l}\mathrm{TT}<2.5 \text { percentile } \\
\text { of the healthy old } \\
\text { men }\end{array}$ & 17 \\
\hline \multicolumn{6}{|c|}{ Miscellaneous diseases } \\
\hline $\begin{array}{l}\text { Schatzl et al., } 2000 \\
\text { [81] }\end{array}$ & $\begin{array}{l}\text { Austria/October } \\
1998 \text { to May } 1999\end{array}$ & Medical clinic & $\begin{array}{l}312 \text { men with lower } \\
\text { urinary tract } \\
\text { symptoms }\end{array}$ & $\mathrm{T}<3 \mathrm{ng} / \mathrm{mL}$ & 22.1 \\
\hline $\begin{array}{l}\text { Rhoden et al., } 2005 \\
\text { [77] }\end{array}$ & $\begin{array}{c}\text { Brazil/January } 2000 \\
\text { to December } 2001\end{array}$ & Medical clinic & $\begin{array}{c}746 \text { urological } \\
\text { patients }\end{array}$ & $\mathrm{TT}<13.87 \mathrm{nmol} / \mathrm{L}$ & 24.5 \\
\hline $\begin{array}{l}\text { Taddesse et al., } 2012 \\
\text { [86] }\end{array}$ & $\begin{array}{l}\text { US/September } 21 \text { to } \\
\text { December 21, } 2010\end{array}$ & Medical clinic & $\begin{array}{l}34 \text { men with sickle } \\
\text { cell }\end{array}$ & $\mathrm{T} \leq 250 \mathrm{ng} / \mathrm{dL}$ & 24 \\
\hline $\begin{array}{l}\text { Okun et al., } 2004 \\
\text { [72] }\end{array}$ & US/not specified & Chart review & $\begin{array}{l}118 \text { men with } \mathrm{PD}+ \\
\text { AD }\end{array}$ & $\begin{array}{l}\mathrm{PD}+\mathrm{T}<325 \mathrm{ng} / \mathrm{mL} \\
\mathrm{AD}+\mathrm{T}<325 \mathrm{ng} / \mathrm{mL}\end{array}$ & $\begin{array}{l}35 \\
38\end{array}$ \\
\hline $\begin{array}{l}\text { Moreno et al., } 2009 \\
{[69]}\end{array}$ & $\begin{array}{c}\text { US/January } 2006 \text { to } \\
\text { January } 2008\end{array}$ & Medical chart review & $\begin{array}{c}121 \text { men with } \\
\text { Peyronie's disease }\end{array}$ & $\mathrm{TT}<300 \mathrm{ng} / \mathrm{dL}$ & 29.5 \\
\hline
\end{tabular}

CHD: chronic heart failure; CKD: chronic kidney disease; ED: erectile dysfunction; ESRD: end stage renal disease; MetS: metabolic syndrome; RA: rheumatoid arthritis; T2DM: type 2 diabetes mellitus; PD: Parkinson's disease; AD: Alzheimer's disease.

hypogonadism and prevalence was also apparent among the primary care-/health screening-based studies (12-39\%).

Prevalence also varied by age. Hypogonadism was consistently found to increase with age for studies reporting data by age (REF), with one study citing an increase of $17 \%$ (95\% CI, $1.08-1.27$ ) in risk of hypogonadism, for every 10 -year increase in age [13]. One study that had a considerably older cohort (mean age of 75.8 years) [6] reported the highest prevalence (31.2\%) among community-based studies. It was estimated in a 2007 publication that there were 4.7 million American men aging 30-79 years with symptomatic androgen deficiency [3]. It was projected that there will be as many as 6.5 million American men aging 30-79 years with symptomatic hypogonadism by 2025 , an increase of $38 \%$ from the year 2000 population estimates, due to an aging population [3].

Despite the significant prevalence of hypogonadism, data suggest that the vast majority of hypogonadal men are not receiving treatment for this condition [13, 98]. Reasons for this low treatment proportion have not been definitively determined; however, contributing factors may include inadequate knowledge base among physicians regarding hypogonadism and uncertainty regarding diagnostic criteria. The main indication for testosterone replacement therapy is sexual dysfunction with symptoms of reduced libido and/or erectile dysfunction. This may not be an issue to some men and therefore they are not treated or subjects may decline therapy. Also, individuals may not be able to afford therapy in countries where people have to pay for their prescriptions and healthcare. Furthermore, with the lack of long-term placebo-controlled safety studies, some clinicians may have concerns especially in the older man in regard to risks of prostate cancer and cardiovascular disease, even though there is limited evidence from current published studies or meta-analyses. The only study which did show a possible relationship between testosterone replacement therapy and cardiovascular events was the TOM trial [99]. However, this study involved the administration of larger testosterone doses than those used in normal clinical practice and was not sufficiently powered to detect an increase in cardiovascular events. A similar trial where routine doses of testosterone were used did not detect any increase in cardiovascular events [100].

Results of this review highlight the fact that there is no standardized definition used in the hypogonadism epidemiologic research. The hypogonadism literature reports wide-ranging prevalence based not only on the different populations or subpopulations studied, but also on the use of diverse biochemical cut points or varied choices of symptoms. The actual prevalence of disease that a clinician will treat in his or her office may in fact be higher than what we found in this review, as clinicians may use a more liberal definition of hypogonadism to base their treatment decisions on.

The studies in this review represented a wide geographic distribution with samples from USA, Europe, Asia, and South America. The prevalence of hypogonadism may vary by geographical location, as recently demonstrated in a large $(n=5003)$ international cohort of older men $(>65$ years of age) showing important geographical differences in concentrations of $\mathrm{T}$ [101]. However, differences in patient populations and operational definitions used across studies made it difficult to interpret the findings based on geography in the current review.

We can expect approximately 12 new cases of hypogonadism per 1,000 person-years [2, 5] and approximately 481,000 new cases of hypogonadism per year in US men aged 40-69 years [2]. It has been projected that there will be as 
TABle 4: Prevalence of hypogonadism by age.

\begin{tabular}{|c|c|c|c|c|c|c|c|}
\hline $\begin{array}{l}\text { Author and year } \\
\text { (study name) }\end{array}$ & $\begin{array}{l}\text { Country and } \\
\text { years of data } \\
\text { collected }\end{array}$ & Study design & $N$ & $\begin{array}{l}\text { Age, mean }(\mathrm{SD}) \text {, and } \\
\text { range }\end{array}$ & $\begin{array}{l}\text { Definition of } \\
\text { hypogonadism used }\end{array}$ & \multicolumn{2}{|c|}{$\begin{array}{l}\text { Prevalence, per age } \\
\text { group, years }(\%)\end{array}$} \\
\hline \multirow{7}{*}{$\begin{array}{l}\text { Harman et al., } 2001 \\
\text { (BLSA) [17] }\end{array}$} & \multirow{7}{*}{$\begin{array}{l}\text { USA, } 40 \text {-year } \\
\text { period }\end{array}$} & \multirow{7}{*}{ Population-based } & \multirow{7}{*}{890} & \multirow{7}{*}{53.8 (16.0), NR } & \multirow{7}{*}{$\begin{array}{l}\mathrm{TT}<325 \mathrm{ng} / \mathrm{dL} \\
\text { OR } \\
\mathrm{FT}<0.153 \mathrm{nmol} / \mathrm{L}\end{array}$} & $20-29$ & $<5$ \\
\hline & & & & & & $30-39$ & $<5$ \\
\hline & & & & & & $40-49$ & $<10$ \\
\hline & & & & & & $50-59$ & 12 \\
\hline & & & & & & $60-69$ & 19 \\
\hline & & & & & & $70-79$ & 28 \\
\hline & & & & & & $>80$ & $49^{\mathrm{a}}$ \\
\hline \multirow{4}{*}{$\begin{array}{l}\text { Wu et al., } 2010 \text { [19] } \\
\text { (EMAS) }\end{array}$} & \multirow{4}{*}{$\begin{array}{l}8 \text { European } \\
\text { countries, } \\
2003-2005\end{array}$} & \multirow{4}{*}{ Population-based } & \multirow{4}{*}{2966} & \multirow{4}{*}{59.15 (10.82), 40-79 } & \multirow{4}{*}{$\begin{array}{l}\mathrm{TT}<317 \mathrm{ng} / \mathrm{dL}+ \\
\mathrm{FT}<220 \mathrm{pmol} / \mathrm{L}+ \\
\geq 3 \text { sexual symptoms }\end{array}$} & $40-49$ & 0.1 \\
\hline & & & & & & $50-59$ & 0.6 \\
\hline & & & & & & $60-69$ & 3.2 \\
\hline & & & & & & $70-79$ & $5.1^{\mathrm{b}}$ \\
\hline \multirow{5}{*}{$\begin{array}{l}\text { Araujo et al., } 2007 \\
\text { [3] (BACH) }\end{array}$} & \multirow{5}{*}{ USA, 2002-2005 } & \multirow{5}{*}{ Population-based } & \multirow{5}{*}{1475} & \multirow{5}{*}{$47.3(12.5)$} & \multirow{5}{*}{$\begin{array}{l}\mathrm{TT}<300 \mathrm{ng} / \mathrm{dL}+ \\
\mathrm{FT}<5 \mathrm{ng} / \mathrm{dL}+ \\
\geq 1 \text { specific symptom or } \\
\geq 2 \text { nonspecific } \\
\text { symptoms }^{\dagger}\end{array}$} & $30-39$ & 1.1 \\
\hline & & & & & & $40-49$ & 4.1 \\
\hline & & & & & & $50-59$ & 8.1 \\
\hline & & & & & & $60-69$ & 9.1 \\
\hline & & & & & & $70-79$ & $22.1^{\mathrm{c}}$ \\
\hline $\begin{array}{l}\text { Araujo et al., } 2004 \\
\text { [2] (MMAS) }\end{array}$ & USA, 1987-1997 & Population-based & 1691 & NR (NR), 40-70 & $\begin{array}{l}\mathrm{TT}<200 \mathrm{ng} / \mathrm{dL} \\
+\geq 3 \text { symptoms }{ }^{\dagger \dagger} \\
\text { OR: } \\
\text { TT } 200-400 \mathrm{ng} / \mathrm{dL}+ \\
\text { FT }<8.91 \mathrm{ng} / \mathrm{dL}+ \\
\geq 3 \text { symptoms }^{\dagger \dagger}\end{array}$ & $\begin{array}{l}40-49 \\
50-59 \\
60-70\end{array}$ & $\begin{array}{r}4.1 \\
4.5^{\mathrm{d}} \\
9.4^{\mathrm{d}}\end{array}$ \\
\hline \multirow{4}{*}{ Li et al., 2005 [18] } & \multirow{4}{*}{ China, 2002-2003 } & \multirow{4}{*}{$\begin{array}{l}\text { Population-based } \\
\text { study }\end{array}$} & \multirow{4}{*}{1080} & \multirow{4}{*}{ NR (NR), 20->70 } & \multirow{4}{*}{$\mathrm{cFT}<0.3 \mathrm{nmol} / \mathrm{L}$} & $40-49$ & 13.0 \\
\hline & & & & & & $50-59$ & 31.8 \\
\hline & & & & & & $60-69$ & 30.1 \\
\hline & & & & & & $>70$ & $46.7^{\mathrm{e}}$ \\
\hline \multirow{4}{*}{$\begin{array}{l}\text { Wong et al., } 2006 \\
{[8]}\end{array}$} & & & & & $\mathrm{TT}<200 \mathrm{ng} / \mathrm{dL}+$ & $45-49$ & 8.2 \\
\hline & China, 2003-2004 & $\begin{array}{l}\text { Community- } \\
\text { based }\end{array}$ & 252 & 54 (NR), 45-64 & $\begin{array}{l}\geq 3 \text { of } 7 \text { symptoms } \\
\text { OR: }\end{array}$ & $50-54$ & 6.3 \\
\hline & & & & & TT $200-400 \mathrm{ng} / \mathrm{dL}+$ & $55-59$ & 10 \\
\hline & & & & & $\begin{array}{l}\mathrm{FT}<0.3093 \mathrm{nmol} / \mathrm{L}+ \\
\geq 3 \text { of } 7 \text { symptoms }\end{array}$ & $60-65$ & $16.7^{\mathrm{f}}$ \\
\hline & & & & & & $45-54$ & 34 \\
\hline & & & & & & $55-64$ & 40.2 \\
\hline & & & & & & $65-74$ & 39.9 \\
\hline Mulligan et al., & USA, 2003-2004 & Primary care & 2162 & 60.5 (10.33), 45-96 & $\mathrm{TT}<300 \mathrm{ng} / \mathrm{dL}$ & $75-84$ & 45.5 \\
\hline 2006 [13] (HIM) & & & & & & $85+$ & 50 \\
\hline & & & & & & $50-54$ & 6.33 \\
\hline & & & & & & $55-59$ & 10 \\
\hline & & & & & & $60-64$ & $16.67^{\mathrm{g}}$ \\
\hline & & & & & & $40-49$ & 16.5 \\
\hline Liu et al., 2009 [12] & Taiwan, & $\begin{array}{l}\text { Health screening } \\
\text { clinic in a }\end{array}$ & 734 & $57.4(6.7), 43-87$ & $\mathrm{TT}<300 \mathrm{ng} / \mathrm{dL}$ & $50-59$ & 23 \\
\hline & $2007-2008$ & medical center & & & & $60-69$ & 28.9 \\
\hline & & & & & & $\geq 70$ & $37.2^{\mathrm{h}}$ \\
\hline
\end{tabular}


TABLE 4: Continued.

\begin{tabular}{|c|c|c|c|c|c|c|c|}
\hline $\begin{array}{l}\text { Author and year } \\
\text { (study name) }\end{array}$ & $\begin{array}{c}\text { Country and } \\
\text { years of data } \\
\text { collected }\end{array}$ & Study design & $N$ & $\begin{array}{c}\text { Age, mean }(\mathrm{SD}) \text {, and } \\
\text { range }\end{array}$ & $\begin{array}{l}\text { Definition of } \\
\text { hypogonadism used }\end{array}$ & \multicolumn{2}{|c|}{$\begin{array}{l}\text { Prevalence, per age } \\
\text { group, years (\%) }\end{array}$} \\
\hline \multirow{3}{*}{$\begin{array}{l}\text { Di Luigi et al., } 2010 \\
\text { [16] }\end{array}$} & \multirow{3}{*}{ Italy, NR } & \multirow{3}{*}{$\begin{array}{l}\text { Health screening } \\
\text { clinic }^{*}\end{array}$} & \multirow{3}{*}{183} & \multirow{3}{*}{$\begin{array}{l}61.9 \pm 7.5 \text { years } \\
\text { (range } 50-75)\end{array}$} & \multirow{3}{*}{$\mathrm{TT}<230 \mathrm{ng} / \mathrm{dL}$} & $50-59$ & 5.4 \\
\hline & & & & & & $60-69$ & 10 \\
\hline & & & & & & $70-79$ & $27.5^{\mathrm{i}}$ \\
\hline $\begin{array}{l}\text { Blümel et al., } 2009 \\
\text { [9] }\end{array}$ & Chile, NR & $\begin{array}{c}\text { Primary } \\
\text { care-based } \\
\text { (health services } \\
\text { center) }\end{array}$ & 96 & $\begin{array}{c}55.1 \pm 12.0 \text { years } \\
\text { (range } 40-85 \text { years) }\end{array}$ & $\begin{array}{l}\mathrm{BT}<198.4 \mathrm{ng} / \mathrm{dL}+ \\
\text { symptoms }^{* *}\end{array}$ & $\begin{array}{c}40-54 \\
55-69 \\
\geq 70\end{array}$ & $\begin{array}{l}17.9 \\
29.2 \\
66.7^{\prime}\end{array}$ \\
\hline
\end{tabular}

TT: $200 \mathrm{ng} / \mathrm{dL}=6.94 \mathrm{nmol} / \mathrm{L} ; 230 \mathrm{ng} / \mathrm{dL}=8 \mathrm{nmol} / \mathrm{L} ; 300 \mathrm{ng} / \mathrm{dL}=10.4 \mathrm{nmol} / \mathrm{L} ; 317 \mathrm{ng} / \mathrm{dL}=11 \mathrm{nmol} / \mathrm{L} ; 325 \mathrm{ng} / \mathrm{dL}=11.3 \mathrm{nmol} / \mathrm{L} ; 350 \mathrm{ng} / \mathrm{dL}=12 \mathrm{nmol} / \mathrm{L} ; \mathrm{FT}: 5$ $\mathrm{ng} / \mathrm{dL}=0.17 \mathrm{nmol} / \mathrm{L} ; 8.9 \mathrm{ng} / \mathrm{dL}=0.3092 \mathrm{nmoL} / \mathrm{L}$.

ADAM: androgen deficiency in the aging male; BACH: Boston Area Community Health; BT: bioavailable testosterone; cFT: calculated free testosterone; EMAS: European Male Aging Study; HIM: hypogonadism in males; MMAS: Massachusetts Male Aging Study; NR: not reported; SHIP: Study of Health in Pomerania; $\mathrm{TT}=$ total testosterone.

${ }^{a}$ No statistical test done; ${ }^{b}$ no statistical test done; ${ }^{c}$ all pairwise comparisons, $P<0.05$ over 70 versus all other age groups; ${ }^{\mathrm{d}} P$ values for trend $<0.001 ;{ }^{\mathrm{e}}$ chi square $P<0.000 ;{ }^{\mathrm{f}}$ not significant; ${ }^{\mathrm{g}}$ no statistical test done; ${ }^{\mathrm{h}} P<0.019$ for trend; ${ }^{\mathrm{i}} P<0.01$ versus age $50-59$ and age $60-69 ;{ }^{\mathrm{j}} P$ value for trend $P<0.0004$.

${ }^{\dagger}$ Specific symptoms include low libido, erective dysfunction, or osteoporosis; the nonspecific symptoms include sleep disturbance, depressed mood, lethargy, or low physical performance.

${ }^{\dagger \dagger}$ Symptoms include loss of libido, erectile dysfunction, depression, lethargy, inability to concentrate, sleep disturbance, irritability, and depressed mood.

" Specific signs/symptoms are low libido, erectile dysfunction, or osteoporosis.

${ }^{*}$ To evaluate risks associated with participation in sports in a group of healthy athletic men.

*** Positive answer to items 1 or 7 , or any 3 other questions on the ADAM questionnaire.

many as 6.5 million US men aging 30-79 years with symptomatic AD by 2025 [3], an increase of $38 \%$ from the year 2000 population estimates, due to an aging population. While there is no conclusive evidence from this review that the prevalence of hypogonadism in the general population is increasing over time, there is evidence that the prevalence of hypogonadism does increase with age and with certain comorbidities such as T2DM and obesity, all of which are increasing in the general population. It follows that there may well be an increasing proportion of older men who will benefit from treatment for hypogonadism. As the proportion of the world's population in the older ages continues to increase, the importance of studying, diagnosing, and treating male hypogonadism will also rise.

The mainstay treatment of low testosterone is testosterone replacement therapy (TRT), although there is indication that modification of diet and exercise regimes have the ability to improve testosterone levels [99]. Despite the significant prevalence of hypogonadism, self-reported data suggest that the vast majority of hypogonadal men are not receiving TRT treatment for this condition [13, 98]. Reasons for this low treatment proportion have not been definitively determined; however, contributing factors may include inadequate knowledge base among physicians regarding hypogonadism and uncertainty regarding diagnostic criteria. The main indication for TRT is sexual dysfunction, which, for some men, may not affect their daily lives, and therefore they choose not to be treated. Also, individuals may not be able to afford therapy in countries where people have to pay for their prescriptions and healthcare.

Another impeding factor for prescribing TRT may be a concern over the potential risk of cardiovascular disease and prostate cancer. The safety of TRT lacks examination in large, prospective, and adequately powered controlled trials [100].
Smaller studies have been published, some suggesting an association between testosterone replacement therapy and cardiovascular adverse events [100]. The TOM trial was terminated early due to an increased frequency of cardiovascular events in the men treated with testosterone [102]. However, this study involved the administration of larger testosterone doses than those used in normal clinical practice and was not sufficiently powered to detect an increase in cardiovascular events. A similar trial, which used routine doses of testosterone, did not detect any increase in cardiovascular events [103]. To address the question of safety and in lieu of long term, prospective safety data, the Endocrine Society Task Force on Testosterone Use in Adult Men commissioned a meta-analysis of randomized and observational studies with long-term follow-up in testosterone therapy in 2010. The analyses of 51 studies found no significant effect of TRT on mortality, prostate, or cardiovascular outcomes [100]. More recently, a retrospective database study of 8709 male veterans who underwent coronary angiography between 2005 and 2011 reported an increased risk (absolute risk increase: $5.8 \%$ (95\% CI: $-1.4 \%$ to $13.1 \%)$ ) of experiencing an adverse cardiovascular event (all-cause mortality, MI, or ischemic stroke) among TRT versus nontreated men at 3 years after coronary angiography [104]. Although this study garnered media attention, it is limited by serious methodological concerns which put the results in question.

4.1. Limitations. A general limitation in hypogonadism research is the lack of a standardized operational definition of hypogonadism. Recently, professional societies have proposed operational definitions which include criteria of specific signs and symptoms plus biochemical low TT, typically defined as TT $<300 \mathrm{ng} / \mathrm{dL}(<10.4 \mathrm{nmol} / \mathrm{L})$ [1]. However, the literature is confounded by numerous studies using 
a variety of different definitions: some include sign and symptom criteria and others do not; biochemical TT thresholds used range from $<8 \mathrm{nmol} / \mathrm{L}$ to $<12 \mathrm{nmol} / \mathrm{L}$, others use an FT criterion and no TT (as men with normal TT may be quite symptomatic in clinical due to low FT), and others use a TT and FT criterion. The varying definitions used make comparisons between studies difficult.

The proportions of patients receiving $\mathrm{T}$ therapy in the population were obtained from one population-based study and one primary care-based study, both of which reported on the number of participants who were receiving $\mathrm{T}$ therapy. Medication use, including use of T therapy, was assessed by patient self-report in the studies. Self-report of medication use is subjective and is at risk of both reporting bias and measurement bias. A more accurate measurement of treatment proportions could be obtained from chart review or commercial claims database study and may be an interesting objective for a future study.

As with all systematic reviews, publication bias, which can lead to bias if only positive studies or studies with a statistically significant difference are published, is an issue in this review. Another limitation of this review is that a quality appraisal of the individual studies was not conducted. Although methodological aspects of the individual studies, which may have influenced outcomes or comparisons between studies, were considered and commented on during the assessment of the evidence, a systematic quality assessment of the studies was not carried out.

\section{Conclusions}

This literature review suggests that there is potentially a significant burden of hypogonadism in the general population. The burden of hypogonadism increases with age and in the presence of certain clinical conditions, such as T2DM and obesity. Data suggested that the vast majority of hypogonadal men in the general population are not receiving treatment. This may be the result of underdiagnosis of the disease, due to lack of awareness by patients and/or physicians and the irregularities surrounding the diagnostic criteria.

\section{Disclosure}

The authors met criteria for authorship as recommended by the International Committee of Medical Journal Editors and are fully responsible for all content and editorial decisions and were involved in all stages of paper development. This original paper has not been previously presented in whole by the authors. A portion of the information has been presented as a poster entitled "The prevalence, incidence and treatment rates of hypogonadism in men across geographies: a systematic review of the literature" at the ISPOR, May 18-22, 2013, New Orleans, LA.

\section{Conflict of Interests}

Victoria Zarotsky and Wendy Carman are employees of Optum and were contracted by Merck \& Co., Inc., to conduct this study. Donna Coffin is employee of Boolean Research Consulting Services who was contracted by Optum. Ming-Yi Huang is a Postdoctoral Fellow funded by Merck \& Co., Inc. Puneet K. Singhal is employed by Merck \& Co., Inc. T. Hugh Jones has received research grants from BayerHealthcare, UK, and honoraria for educational lectures and/or advisory boards from BayerHealthcare, Clarus, Lilly, Merck, and Prostrakan. T. Hugh Jones and Abraham Morgentaler have served as Consultants to Merck \& Co., Inc., for this study.

\section{Acknowledgments}

This study was conducted by Optum located in Eden Prairie, Minnesota, USA, and was funded by Merck \& Co., Inc., West Point, PA. This study was supported by Merck \& Co., Inc.

\section{References}

[1] S. Bhasin, G. R. Cunningham, F. J. Hayes et al., "Testosterone therapy in men with androgen deficiency syndromes: an endocrine society clinical practice guideline," The Journal of Clinical Endocrinology \& Metabolism, vol. 95, no. 6, pp. 25362559, 2010.

[2] A. B. Araujo, A. B. O’Donnell, D. J. Brambilla et al., "Prevalence and incidence of androgen deficiency in middle-aged and older men: Estimates from the Massachusetts male aging study," Journal of Clinical Endocrinology and Metabolism, vol. 89, no. 12, pp. 5920-5926, 2004.

[3] A. B. Araujo, G. R. Esche, V. Kupelian et al., "Prevalence of symptomatic androgen deficiency in men," Journal of Clinical Endocrinology and Metabolism, vol. 92, no. 11, pp. 4241-4247, 2007.

[4] A. Tajar, I. T. Huhtaniemi, T. W. O'Neill et al., "Characteristics of androgen deficiency in Late-onset hypogonadism: results from the European Male Aging study (EMAS)," Journal of Clinical Endocrinology and Metabolism, vol. 97, no. 5, pp. 1508-1516, 2012.

[5] R. Haring, T. Ittermann, H. Völzke et al., "Prevalence, incidence and risk factors of testosterone deficiency in a population-based cohort of men: results from the study of health in Pomerania," Aging Male, vol. 13, no. 4, pp. 247-257, 2010.

[6] A. Ponholzer, S. Madersbacher, M. Rauchenwald, S. Jungwirth, P. Fischer, and K. Tragl, "Vascular risk factors and their association to serum androgen levels in a population-based cohort of 75-year-old men over 5 years: results of the VITA study," World Journal of Urology, vol. 28, no. 2, pp. 209-214, 2010.

[7] E. M. Khoo, H. M. Tan, and W. Y. Low, "Erectile dysfunction and comorbidities in aging men: an urban cross-sectional study in Malaysia," Journal of Sexual Medicine, vol. 5, no. 12, pp. 29252934, 2008.

[8] S. Y. S. Wong, D. C. C. Chan, A. Hong, and J. Woo, "Prevalence of and risk factors for androgen deficiency in middle-aged men in Hong Kong," Metabolism: Clinical and Experimental, vol. 55, no. 11, pp. 1488-1494, 2006.

[9] J. E. Blümel, P. Chedraui, S. A. Gili, A. Navarro, K. Valenzuela, and S. Vallejo, "Is the Androgen Deficiency of Aging Men (ADAM) questionnaire useful for the screening of partial androgenic deficiency of aging men?" Maturitas, vol. 63, no. 4, pp. 365-368, 2009.

[10] L. di Luigi, P. Sgrò, V. Fierro et al., "Prevalence of undiagnosed testosterone deficiency in aging athletes: does exercise training 
influence the symptoms of male hypogonadism?" Journal of Sexual Medicine, vol. 7, no. 7, pp. 2591-2601, 2010.

[11] A. Goel, R. J. Sinha, D. Dalela, S. Sankhwar, and V. Singh, "Andropause in Indian men a preliminary cross-sectional study," Urology Journal, vol. 6, no. 1, pp. 40-46, 2009.

[12] C.-C. Liu, W.-J. Wu, Y.-C. Lee et al., "The prevalence of and risk factors for androgen deficiency in aging Taiwanese men," Journal of Sexual Medicine, vol. 6, no. 4, pp. 936-946, 2009.

[13] T. Mulligan, M. F. Frick, Q. C. Zuraw, A. Stemhagen, and C. McWhirter, "Prevalence of hypogonadism in males aged at least 45 years: The HIM study," International Journal of Clinical Practice, vol. 60, no. 7, pp. 762-769, 2006.

[14] A. N. Júnior, S. dos Santos Szelbracikowski, A. C. Nardi, and J. C. de Almeida, "Age-related testosterone decline in a Brazilian cohort of healthy military men," International Brazilian Journal of Urology, vol. 37, no. 5, pp. 591-597, 2011.

[15] H. J. Schneider, C. Sievers, J. Klotsche et al., "Prevalence of low male testosterone levels in primary care in Germany: crosssectional results from the DETECT study," Clinical Endocrinology, vol. 70, no. 3, pp. 446-454, 2009.

[16] L. Di Luigi, P. Sgrò, V. Fierro et al., "Prevalence of undiagnosed testosterone deficiency in aging athletes: does exercise training influence the symptoms of male hypogonadism?" Journal of Sexual Medicine, vol. 7, no. 7, pp. 2591-2601, 2010.

[17] S. M. Harman, E. J. Metter, J. D. Tobin, J. Pearson, and M. R. Blackman, "Longitudinal effects of aging on serum total and free testosterone levels in healthy men," Journal of Clinical Endocrinology and Metabolism, vol. 86, no. 2, pp. 724-731, 2001.

[18] J.-Y. Li, X.-Y. Li, M. Li et al., "Decline of serum levels of free testosterone in aging healthy Chinese men," Aging Male, vol. 8, no. 3-4, pp. 203-206, 2005.

[19] F. C. W. Wu, A. Tajar, J. M. Beynon et al., "Identification of lateonset hypogonadism in middle-aged and elderly men," The New England Journal of Medicine, vol. 363, no. 2, pp. 123-135, 2010.

[20] A. Abbasi, D. E. Mattson, M. Cuisinier et al., "Hyposomatomedinemia and hypogonadism in hemiplegic men who live in nursing homes," Archives of Physical Medicine and Rehabilitation, vol. 75, no. 5, pp. 594-599, 1994.

[21] F. Albaaj, M. Sivalingham, P. Haynes et al., "Prevalence of hypogonadism in male patients with renal failure," Postgraduate Medical Journal, vol. 82, no. 972, pp. 693-696, 2006.

[22] C. A. Allan, B. J. Strauss, H. G. Burger, E. A. Forbes, and R. I. McLachlan, "The association between obesity and the diagnosis of androgen deficiency in symptomatic ageing men," Medical Journal of Australia, vol. 185, no. 8, pp. 424-427, 2006.

[23] S. G. Anderson, A. Heald, N. Younger et al., "Screening for hypogonadism in diabetes 2008/9: results from the Cheshire Primary Care cohort," Primary Care Diabetes, vol. 6, no. 2, pp. 143-148, 2012.

[24] M. Arafa, W. Zohdy, S. Aboulsoud, and R. Shamloul, "Prevalence of late-onset hypogonadism in men with type 2 diabetes mellitus," Andrologia, vol. 44, no. 1, pp. 756-763, 2012.

[25] M. Biswas, D. Hampton, R. G. Newcombe, and D. A. Rees, "Total and free testosterone concentrations are strongly influenced by age and central obesity in men with type 1 and type 2 diabetes but correlate weakly with symptoms of androgen deficiency and diabetes-related quality of life," Clinical Endocrinology, vol. 76, no. 5, pp. 665-673, 2012.

[26] T. J. Bunch, D. Abraham, S. Wang, and A. W. Meikle, "Pituitary radiographic abnormalities and clinical correlates of hypogonadism in elderly males presenting with erectile dysfunction," Aging Male, vol. 5, no. 1, pp. 38-46, 2002.
[27] A. D. A. Caldas, A. L. Porto, L. D. C. D. Motta, and L. A. Casulari, "Relationship between insulin and hypogonadism in men with metabolic syndrome," Arquivos Brasileiros de Endocrinologia e Metabologia, vol. 53, no. 8, pp. 1005-1011, 2009.

[28] J. J. Carrero, A. R. Qureshi, A. Nakashima et al., "Prevalence and clinical implications of testosterone deficiency in men with end-stage renal disease," Nephrology Dialysis Transplantation, vol. 26, no. 1, pp. 184-190, 2011.

[29] R. Y. T. Chen, G. A. Wittert, and G. R. Andrews, "Relative androgen deficiency in relation to obesity and metabolic status in older men," Diabetes, Obesity and Metabolism, vol. 8, no. 4, pp. 429-435, 2006.

[30] R. Clapauch, D. J. D. C. Braga, L. P. Marinheiro, S. Buksman, and Y. Schrank, "Risk of late-onset hypogonadism (Andropause) in Brazilian men over 50 years of age with osteoporosis: usefulness of screening questionnaires," Arquivos Brasileiros de Endocrinologia e Metabologia, vol. 52, no. 9, pp. 1439-1447, 2008.

[31] J. Collazos and M. Esteban, "Has prolactin a role in the hypogonadal status of HIV-infected patients?" Journal of the International Association of Physicians in AIDS Care, vol. 8, no. 1, pp. 43-46, 2009.

[32] G. Corona, V. Boddi, F. Lotti et al., "The Relationship of testosterone to prostate-specific antigen in men with sexual dysfunction," Journal of Sexual Medicine, vol. 7, no. 1, part 1, pp. 284-292, 2010.

[33] G. Corona, E. Mannucci, F. Lotti et al., "Impairment of couple relationship in male patients with sexual dysfunction is associated with overt hypogonadism," Journal of Sexual Medicine, vol. 6, no. 9, pp. 2591-2600, 2009.

[34] G. Corona, E. Mannucci, F. Lotti et al., "Pulse pressure, an index of arterial stiffness, is associated with androgen deficiency and impaired penile blood flow in men with ED," Journal of Sexual Medicine, vol. 6, no. 1, pp. 285-293, 2009.

[35] G. Corona, E. Mannucci, L. Petrone et al., "NCEP-ATPIIIdefined metabolic syndrome, type 2 diabetes mellitus, and prevalence of hypogonadism in male patients with sexual dysfunction," Journal of Sexual Medicine, vol. 4, no. 4, part 1, pp. 1038-1045, 2007.

[36] G. Corona, E. Mannucci, L. Petrone et al., "Association of hypogonadism and type II diabetes in men attending an outpatient erectile dysfunction clinic," International Journal of Impotence Research, vol. 18, no. 2, pp. 190-197, 2006.

[37] G. Corona, M. Monami, V. Boddi et al., "Low testosterone is associated with an increased risk of MACE lethality in subjects with erectile dysfunction," Journal of Sexual Medicine, vol. 7, no. 4, pp. 1557-1564, 2010.

[38] N. F. Crum-Cianflone, M. Bavaro, B. Hale et al., "Erectile dysfunction and hypogonadism among men with HIV," AIDS Patient Care and STDs, vol. 21, no. 1, pp. 9-19, 2007.

[39] S. Dhindsa, M. G. Miller, C. L. McWhirter et al., "Testosterone concentrations in diabetic and nondiabetic obese men," Diabetes Care, vol. 33, no. 6, pp. 1186-1192, 2010.

[40] S. Dhindsa, S. Prabhakar, M. Sethi, A. Bandyopadhyay, A. Chaudhuri, and P. Dandona, "Frequent occurrence of hypogonadotropic hypogonadism in type 2 diabetes," Journal of Clinical Endocrinology and Metabolism, vol. 89, no. 11, pp. 5462-5468, 2004.

[41] A. Durga, F. Sepahpanah, M. Regozzi, J. Hastings, and D. A. Crane, "Prevalence of testosterone deficiency after spinal cord injury," $P M$ \& R, vol. 3, no. 10, pp. 929-932, 2011.

[42] H. A. Fink, S. K. Ewing, K. E. Ensrud et al., "Association of testosterone and estradiol deficiency with osteoporosis and 
rapid bone loss in older men," Journal of Clinical Endocrinology and Metabolism, vol. 91, no. 10, pp. 3908-3915, 2006.

[43] S. B. Fleishman, H. Khan, P. Homel et al., "Testosterone levels and quality of life in diverse male patients with cancers unrelated to androgens," Journal of Clinical Oncology, vol. 28, no. 34, pp. 5054-5060, 2010.

[44] A. Florvaag, V. Oberle, M. Fritzenwanger et al., "Testosterone deficiency in male heart failure patients and its effect on endothelial progenitor cells," Aging Male, vol. 15, no. 3, pp. 180186, 2012.

[45] H. K. Ganesh, H. A. V. Sarathi, J. George et al., "Prevalence of hypogonadism in patients with type 2 diabetes mellitus in an Asian Indian study group," Endocrine Practice, vol. 15, no. 6, pp. 513-520, 2009.

[46] D. M. Greenfield, S. J. Walters, R. E. Coleman et al., "Prevalence and consequences of androgen deficiency in young male cancer survivors in a controlled cross-sectional study," Journal of Clinical Endocrinology and Metabolism, vol. 92, no. 9, pp. 34763482, 2007.

[47] M. Grossmann, S. Panagiotopolous, K. Sharpe et al., "Low testosterone and anaemia in men with type 2 diabetes," Clinical Endocrinology, vol. 70, no. 4, pp. 547-553, 2009.

[48] M. Grossmann, M. C. Thomas, S. Panagiotopoulos et al., "Low testosterone levels are common and associated with insulin resistance in men with diabetes," Journal of Clinical Endocrinology and Metabolism, vol. 93, no. 5, pp. 1834-1840, 2008.

[49] A. Guay, A. D. Seftel, and A. Traish, "Hypogonadism in men with erectile dysfunction may be related to a host of chronic illnesses," International Journal of Impotence Research, vol. 22, no. 1, pp. 9-19, 2010.

[50] G. I. Hackett, N. S. Cole, A. A. Deshpande, M. D. Popple, D. Kennedy, and P. Wilkinson, "Biochemical hypogonadism in men with type 2 diabetes in primary care practice," British Journal of Diabetes and Vascular Disease, vol. 9, no. 5, pp. 226231, 2009.

[51] S. Halabi, E. G. Collins, N. Thorevska, M. J. Tobin, and F. Laghi, "Relationship between depressive symptoms and hypogonadism in men with COPD," COPD: Journal of Chronic Obstructive Pulmonary Disease, vol. 8, no. 5, pp. 346-353, 2011.

[52] M. A. Hamidi, A. Heidarzadeh, P. N. Akbari, D. F. Khosravi, and M. Z. Hamidi, "A survey on relative frequency of metabolic syndrome and testosterone deficiency in men with erectile dysfunction," International Urology and Nephrology, vol. 44, no. 3, pp. 667-672, 2012.

[53] J. Hintikka, L. Niskanen, H. Koivumaa-Honkanen et al., "Hypogonadism, decreased sexual desire, and long-term depression in middle-aged men," Journal of Sexual Medicine, vol. 6, no. 7, pp. 2049-2057, 2009.

[54] J. Hofstra, S. Loves, B. van Wageningen, J. Ruinemans-Koerts, I. Janssen, and H. de Boer, "High prevalence of hypogonadotropic hypogonadism in men referred for obesity treatment," Netherlands Journal of Medicine, vol. 66, no. 3, pp. 103-109, 2008.

[55] E. A. Jankowska, B. Biel, J. Majda et al., "Anabolic deficiency in men with chronic heart failure: prevalence and detrimental impact on survival," Circulation, vol. 114, no. 17, pp. 1829-1837, 2006.

[56] D. Kapoor, H. Aldred, S. Clark, K. S. Channer, and T. H. Jones, "Clinical and biochemical assessment of hypogonadism in men with type 2 diabetes: Correlations with bioavailable testosterone and visceral adiposity," Diabetes Care, vol. 30, no. 4, pp. 911-917, 2007.
[57] G. Kirbas, A. Abakay, F. Topcu, A. Kaplan, M. Unlu, and Y. Peker, "Obstructive sleep apnoea, cigarette smoking and serum testosterone levels in a male sleep clinic cohort," Journal of International Medical Research, vol. 35, no. 1, pp. 38-45, 2007.

[58] R. S. Klein, Y. Lo, N. Santoro, and A. S. Dobs, "Androgen levels in older men who have or who are at risk of acquiring HIV infection," Clinical Infectious Diseases, vol. 41, no. 12, pp. 17941803, 2005.

[59] T. S. Köhler, J. Kim, K. Feia et al., "Prevalence of androgen deficiency in men with erectile dysfunction," Urology, vol. 71, no. 4, pp. 693-697, 2008.

[60] J. Kyriazis, I. Tzanakis, K. Stylianou et al., "Low serum testosterone, arterial stiffness and mortality in male haemodialysis patients," Nephrology Dialysis Transplantation, vol. 26, no. 9, pp. 2971-2977, 2011.

[61] S. la Vignera, A. E. Calogero, R. Condorelli, F. Lanzafame, B. Giammusso, and E. Vicari, "Andrological characterization of the patient with diabetes mellitus," Minerva Endocrinologica, vol. 34, no. 1, pp. 1-9, 2009.

[62] D. E. Laaksonen, L. Niskanen, K. Punnonen et al., "The metabolic syndrome and smoking in relation to hypogonadism in middle-aged men: a prospective cohort study," Journal of Clinical Endocrinology and Metabolism, vol. 90, no. 2, pp. 712719, 2005.

[63] J. E. Lackner, I. Märk, G. Schatzl, M. Marberger, and C. Kratzik, "Hypogonadism and androgen deficiency symptoms in testicular cancer survivors," Urology, vol. 69, no. 4, pp. 754-758, 2007.

[64] F. Laghi, A. Antonescu-Turcu, E. Collins et al., "Hypogonadism in men with chronic obstructive pulmonary disease: prevalence and quality of life," The American Journal of Respiratory and Critical Care Medicine, vol. 171, no. 7, pp. 728-733, 2005.

[65] E. Lerchbaum, S. Pilz, B. O. Boehm, T. B. Grammer, B. Obermayer-Pietsch, and W. März, "Combination of low free testosterone and low vitamin D predicts mortality in older men referred for coronary angiography," Clinical Endocrinology, vol. 77, no. 3, pp. 475-483, 2012.

[66] A. A. Makhlouf, M. A. Mohamed, A. D. Seftel, and C. Neiderberger, "Hypogonadism is associated with overt depression symptoms in men with erectile dysfunction," International Journal of Impotence Research, vol. 20, no. 2, pp. 157-161, 2008.

[67] C. J. Malkin, P. J. Pugh, P. D. Morris, S. Asif, T. H. Jones, and K. S. Channer, "Low serum testosterone and increased mortality in men with coronary heart disease," Heart, vol. 96, no. 22, pp. 1821-1825, 2010.

[68] R. S. McIntyre, D. Mancini, B. S. Eisfeld et al., "Calculated bioavailable testosterone levels and depression in middle-aged men," Psychoneuroendocrinology, vol. 31, no. 9, pp. 1029-1035, 2006.

[69] S. A. Moreno and A. Morgentaler, “Testosterone deficiency and Peyronie's disease: pilot data suggesting a significant relationship," Journal of Sexual Medicine, vol. 6, no. 6, pp. 1729-1735, 2009.

[70] A. O. Ogbera, "Relationship between serum testosterone levels and features of the metabolic syndrome defining criteria in patients with type 2 diabetes mellitus," West African Journal of Medicine, vol. 30, no. 4, pp. 277-281, 2011.

[71] O. A. Ogbera, C. Sonny, F. Olufemi, and A. Wale, "Hypogonadism and subnormal total testosterone levels in men with type 2 diabetes mellitus," Journal of the College of Physicians and Surgeons Pakistan, vol. 21, no. 9, pp. 517-521, 2011. 
[72] M. S. Okun, M. R. DeLong, J. Hanfelt, M. Gearing, and A. Levey, "Plasma testosterone levels in Alzheimer and Parkinson diseases," Neurology, vol. 62, no. 3, pp. 411-413, 2004.

[73] M. S. Okun, W. M. McDonald, and M. R. Delong, "Refractory nonmotor symptoms in male patients with Parkinson disease due to testosterone deficiency: a common unrecognized comorbidity," Archives of Neurology, vol. 59, no. 5, pp. 807-811, 2002.

[74] S. Pellitero, I. Olaizola, A. Alastrue et al., "Hypogonadotropic hypogonadism in morbidly obese males is reversed after bariatric surgery," Obesity Surgery, vol. 22, no. 12, pp. 1835-1842, 2012.

[75] G. Pühse, A. Secker, S. Kemper, L. Hertle, and S. Kliesch, "Testosterone deficiency in testicular germ-cell cancer patients is not influenced by oncological treatment," International Journal of Andrology, vol. 34, no. 5, part 2, pp. e351-e357, 2011.

[76] A. Rajagopal, R. Vassilopoulou-Sellin, J. L. Palmer, G. Kaur, and E. Bruera, "Symptomatic hypogonadism in male survivors of cancer with chronic exposure to opioids," Cancer, vol. 100, no. 4, pp. 851-858, 2004.

[77] E. L. Rhoden, E. P. Ribeiro, C. Teloken, and C. A. V. Souto, "Diabetes mellitus is associated with subnormal serum levels of free testosterone in men," BJU International, vol. 96, no. 6, pp. 867-870, 2005.

[78] P. Rietschel, C. Corcoran, T. Stanley, N. Basgoz, A. Klibanski, and S. Grinspoon, "Prevalence of hypogonadism among men with weight loss related to human immunodeficiency virus infection who were receiving highly active antiretroviral therapy," Clinical Infectious Diseases, vol. 31, no. 5, pp. 1240-1244, 2000.

[79] P. Romerius, O. Ståhl, C. Moëll et al., "Hypogonadism risk in men treated for childhood cancer," Journal of Clinical Endocrinology and Metabolism, vol. 94, no. 11, pp. 4180-4186, 2009.

[80] A. Salonia, A. Gallina, A. Briganti et al., "Preoperative hypogonadism is not an independent predictor of high-risk disease in patients undergoing radical prostatectomy," Cancer, vol. 117, no. 17, pp. 3953-3962, 2011.

[81] G. Schatzl, C. Brössner, S. Schmid et al., "Endocrine status in elderly men with lower urinary tract symptoms: correlation of age, hormonal status, and lower urinary tract function," Urology, vol. 55, no. 3, pp. 397-402, 2000.

[82] G. Schatzl, S. Madersbacher, T. Thurridl et al., "High-grade prostate cancer is associated with low serum testosterone levels," Prostate, vol. 47, no. 1, pp. 52-58, 2001.

[83] S. K. Singh, R. Goyal, and D. D. Pratyush, "Is hypoandrogenemia a component of metabolic syndrome in males?" Experimental and Clinical Endocrinology \& Diabetes, vol. 119, no. 1, pp. 30-35, 2011.

[84] E. Serra, M. Porcu, L. Minerba et al., "High prevalence of male hypogonadism and sexual dysfunction in long-term clinically stable heart transplantation recipients," International Journal of Cardiology, vol. 155, no. 3, pp. 476-477, 2012.

[85] B. Somani, S. Khan, and R. Donat, "Screening for metabolic syndrome and testosterone deficiency in patients with erectile dysfunction: results from the first UK prospective study," $B J U$ International, vol. 106, no. 5, pp. 688-690, 2010.

[86] A. Taddesse, I. L. Woldie, P. Khana et al., "Hypogonadism in patients with sickle cell disease: central or peripheral?" Acta Haematologica, vol. 128, no. 2, pp. 65-68, 2012.
[87] S. K. Talukder, F. Afsana, and S. J. Khan, "Metabolic syndrome in men with sexual dysfunction," Diabetes and Metabolic Syndrome: Clinical Research and Reviews, vol. 4, no. 3, pp. 143-149, 2010.

[88] W. S. Tan, C. J. Ng, E. M. Khoo, W. Low, and H. M. Tan, “The triad of erectile dysfunction, testosterone deficiency syndrome and metabolic syndrome: findings from a multi-ethnic Asian men study (The Subang Men's Health Study)," The Aging Male, vol. 14, no. 4, pp. 231-236, 2011.

[89] B. Tengstrand, K. Carlström, and I. Hafström, "Bioavailable testosterone in men with rheumatoid arthritis-high frequency of hypogonadism," Rheumatology, vol. 41, no. 3, pp. 285-289, 2002.

[90] T. G. Travison, R. Shackelton, A. B. Araujo et al., "The natural history of symptomatic androgen deficiency in men: onset, progression, and spontaneous remission," Journal of the American Geriatrics Society, vol. 56, no. 5, pp. 831-839, 2008.

[91] K. Tromp, J. J. M. Claessens, S. L. Knijnenburg et al., "Reproductive status in adult male long-term survivors of childhood cancer," Human Reproduction, vol. 26, no. 7, pp. 1775-1783, 2011.

[92] G. Wagner, J. G. Rabkin, and R. Rabkin, "Illness stage, concurrent medications, and other correlates of low testosterone in men with HIV illness," Journal of Acquired Immune Deficiency Syndromes and Human Retrovirology, vol. 8, no. 2, pp. 204-207, 1995.

[93] J. T. Wahlstrom, A. Tang, J. Cofrancesco, N. Shah Jr., and A. S. Dobs, "Gonadal hormone levels in injection drug users," Drug and Alcohol Dependence, vol. 60, no. 3, pp. 311-313, 2000.

[94] X.-F. Wang, J.-H. Wang, and J.-Y. Li, "Androgen deficiency in elderly men with systolic chronic heart failure," Journal of Geriatric Cardiology, vol. 7, no. 3-4, pp. 138-142, 2010.

[95] E. Wehr, S. Pilz, B. O. Boehm, W. März, and B. ObermayerPietsch, "Association of vitamin D status with serum androgen levels in men," Clinical Endocrinology, vol. 73, no. 2, pp. 243$248,2010$.

[96] D. M. Wunder, N. A. Bersinger, C. A. Fux et al., "Hypogonadism in HIV-1-infected men is common and does not resolve during antiretroviral therapy," Antiviral Therapy, vol. 12, no. 2, pp. 261265, 2007.

[97] M. I. Yilmaz, A. Sonmez, A. R. Qureshi et al., "Endogenous testosterone, endothelial dysfunction, and cardiovascular events in men with nondialysis chronic kidney disease," Clinical Journal of the American Society of Nephrology, vol. 6, no. 7, pp. 1617-1625, 2011.

[98] S. A. Hall, A. B. Araujo, G. R. Esche et al., "Treatment of symptomatic androgen deficiency: results from the Boston Area Community Health Survey," Archives of Internal Medicine, vol. 168, no. 10, pp. 1070-1076, 2008.

[99] P. Dandona and M. T. Rosenberg, "A practical guide to male hypogonadism in the primary care setting," International Journal of Clinical Practice, vol. 64, no. 6, pp. 682-696, 2010.

[100] M. Fernández-Balsells, M. Murad, M. Lane et al., "Clinical review 1: adverse effects of testosterone therapy in adult men: a systematic review and meta-analysis," Journal of Clinical Endocrinology and Metabolism, vol. 95, no. 6, pp. 2560-2575, 2010.

[101] E. S. Orwoll, C. M. Nielson, F. Labrie et al., "Evidence for geographical and racial variation in serum sex steroid levels in older men," Journal of Clinical Endocrinology and Metabolism, vol. 95, no. 10, pp. E151-E160, 2010. 
[102] S. Basaria, A. D. Coviello, T. G. Travison et al., "Adverse events associated with testosterone administration," The New England Journal of Medicine, vol. 363, no. 2, pp. 109-122, 2010.

[103] U. Srinivas-Shankar, S. A. Roberts, M. J. Connolly et al., "Effects of testosterone on muscle strength, physical function, body composition, and quality of life in intermediate-frail and frail elderly men: a randomized, double-blind, placebo-controlled study," Journal of Clinical Endocrinology and Metabolism, vol. 95, no. 2, pp. 639-650, 2010.

[104] R. Vigen, C. O’Donnell, A. Barón et al., "Association of testosterone therapy with mortality, myocardial infarction, and stroke in men with low testosterone levels," The Journal of the American Medical Association, vol. 310, no. 17, pp. 1829-1836, 2013. 


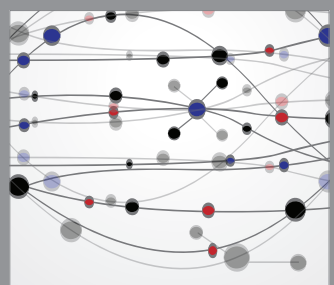

The Scientific World Journal
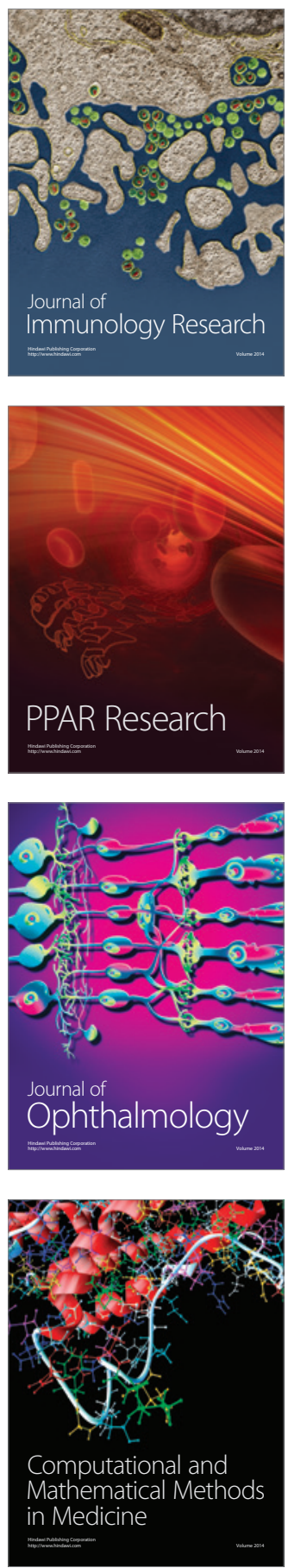

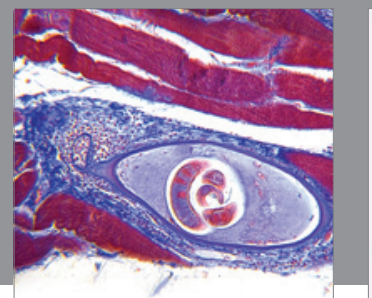

Gastroenterology

Research and Practice
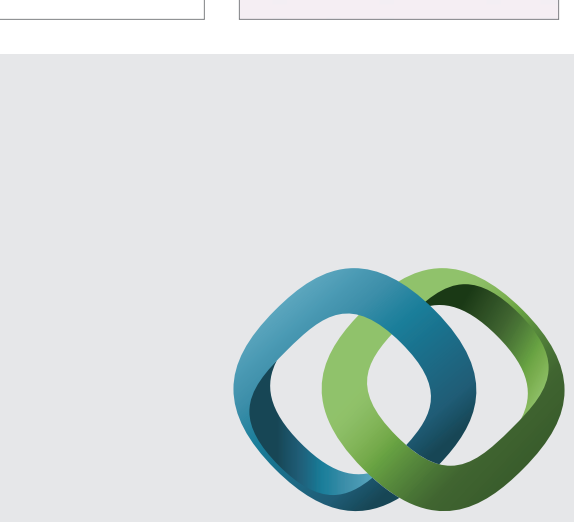

\section{Hindawi}

Submit your manuscripts at

http://www.hindawi.com
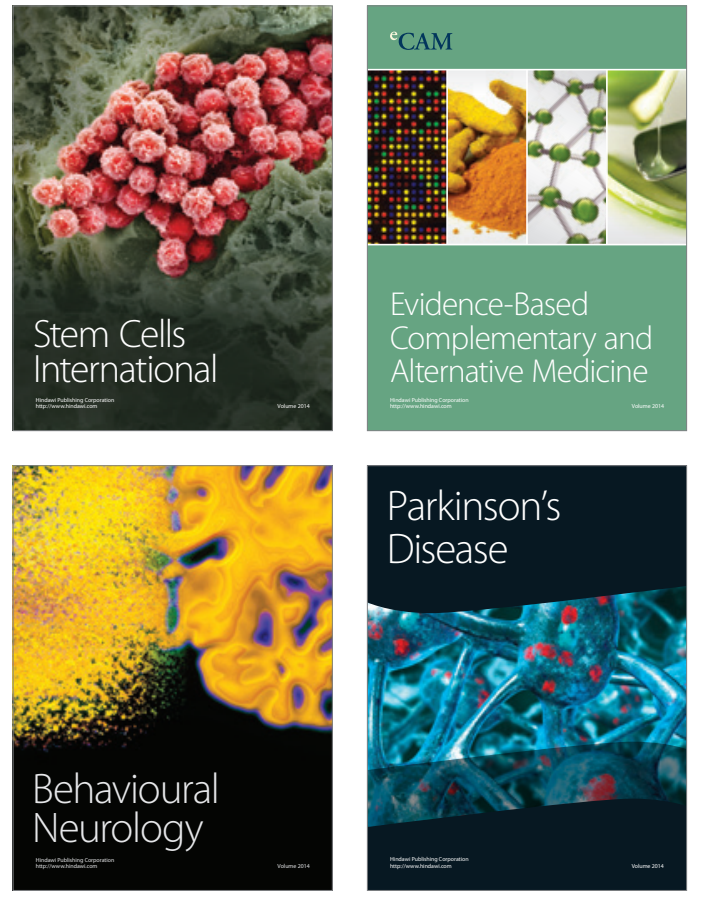
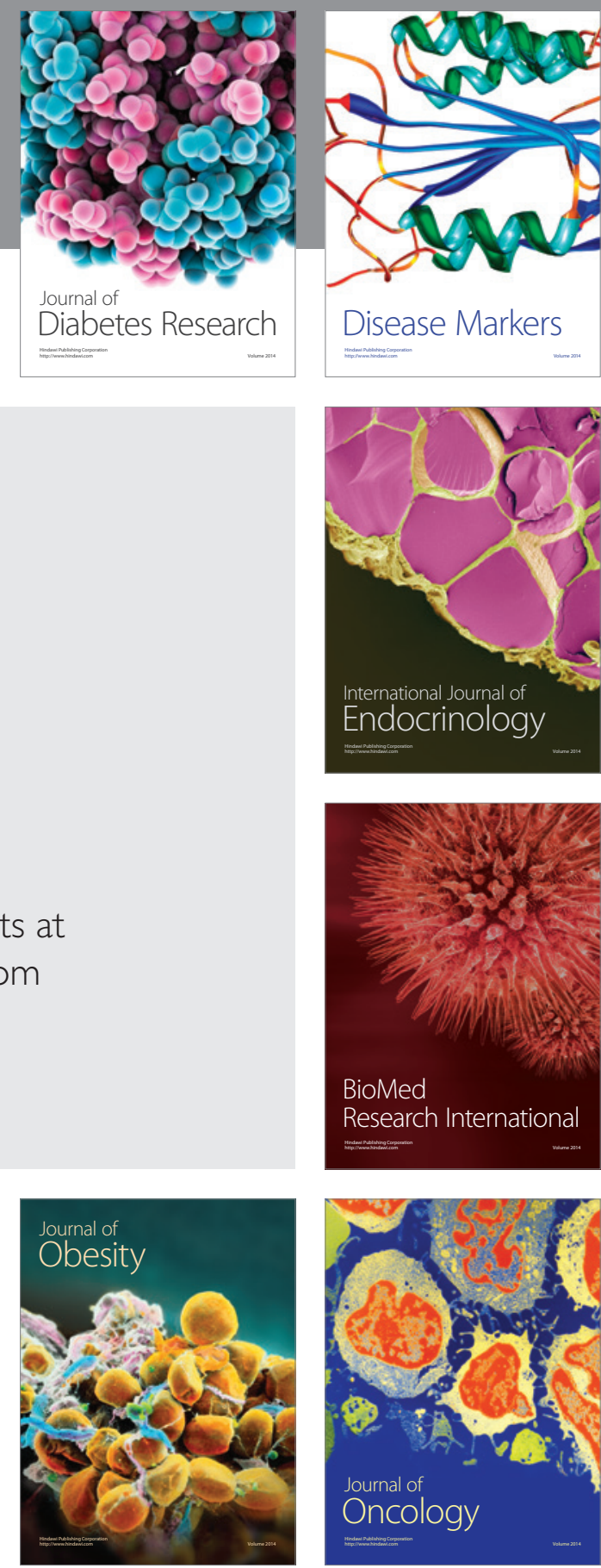

Disease Markers
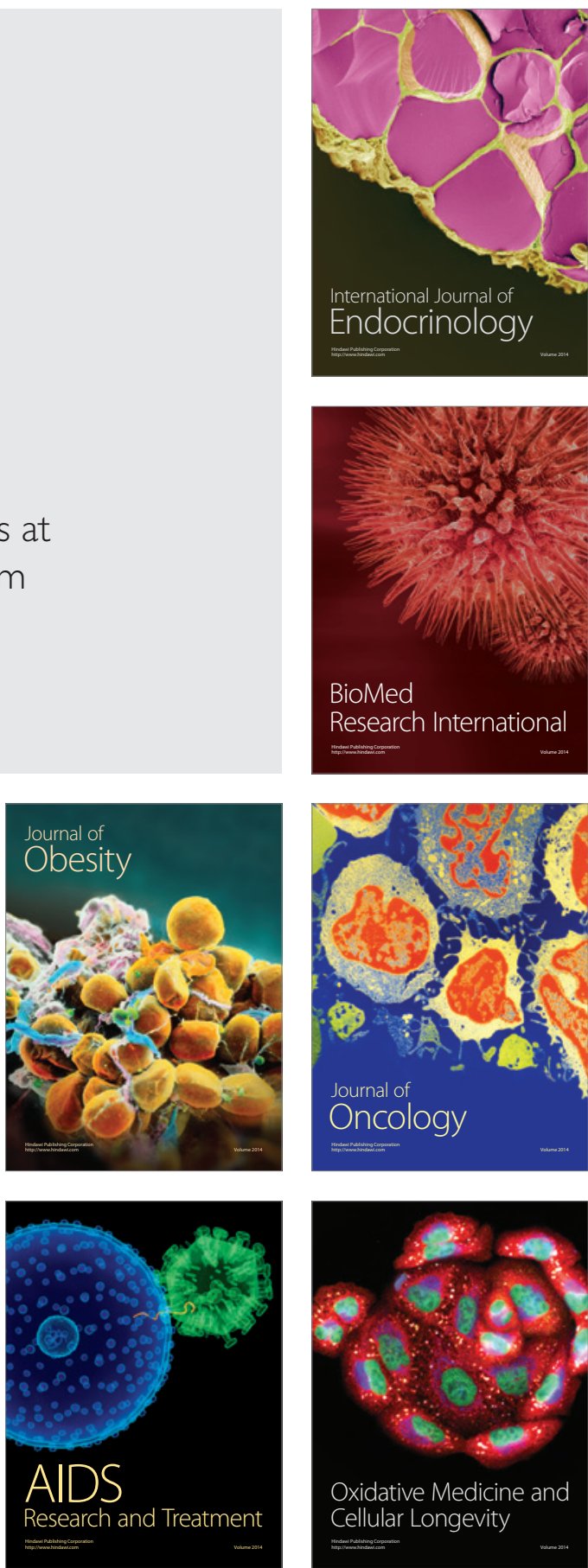